Supporting Information for

\title{
Controlled Assembly Synthesis of Atomically Precise Ultrastable Silver Nanoclusters with Polyoxometalates
}

Kentaro Yonesato, ${ }^{\mathrm{a}}$ Hiroyasu Ito, ${ }^{\mathrm{a}}$ Hiroyuki Itakura, ${ }^{\mathrm{a}}$ Daisuke Yokogawa, ${ }^{\mathrm{b}}$ Takashi Kikuchi, Noritaka Mizuno, ${ }^{\text {a }}$ Kazuya Yamaguchi, ${ }^{*}$, a and Kosuke Suzuki ${ }^{*}, \mathrm{a}, \mathrm{d}$

${ }^{a}$ Department of Applied Chemistry, School of Engineering, The University of Tokyo, 7-3-1 Hongo, Bunkyo-ku, Tokyo 113-8656, Japan.

${ }^{b}$ Graduate School of Arts and Science, The University of Tokyo, 3-8-1 Komaba, Meguro-ku, Tokyo 153-8902, Japan.

${ }^{c}$ Rigaku Corporation, 3-9-12 Matsubaracho, Akishima, Tokyo 196-8666, Japan.

${ }^{\mathrm{d}}$ Precursory Research for Embryonic Science and Technology (PRESTO), Japan Science and Technology Agency (JST), 4-1-8 Honcho, Kawaguchi, Saitama 332-0012, Japan. 
Materials: Acetone, $\mathrm{N}, \mathrm{N}$-dimethylformamide, dichloroethane, diethyl ether, ethyl acetate, tetrachloroethane, and ethylene carbonate were obtained from Kanto Chemicals. All solvents were stored with appropriate molecular sieves. Silver acetate and silver benzoate were purchased from Sigma-Aldrich. $\mathrm{TBA}_{4}\left[\mathrm{SiW}_{9} \mathrm{O}_{28}\left(\mathrm{OCH}_{3}\right)_{6}\right]$ was synthesised according to the reported procedure. ${ }^{\mathrm{S} 1 \mathrm{~S} 2}$

Instruments: Solution-state UV-Vis spectra were measured on a JASCO V-570 spectrometer with $1 \mathrm{~cm}$ quartz cells. Diffuse reflection UV-Vis spectra were measured on a Shimadzu UV-3600 plus. IR spectra were measured on a JASCO FT/IR-4100 spectrometer using KBr disks. ESI-mass spectra were measured on a Waters Xevo G2-XS QTof instrument. ${ }^{1} \mathrm{H}$ and ${ }^{29} \mathrm{Si}$ NMR spectra were measured on a JEOL JNM ECA-500 spectrometer using $5 \mathrm{~mm}$ tubes. Chemical shifts $(\delta)$ were reported using $\mathrm{Si}\left(\mathrm{CH}_{3}\right)_{4}$ as an internal standard. ICP-AES analyses for Si, Ag and W were conducted with a Shimadzu ICPS-8100. Elemental analyses for C, $\mathrm{H}$ and $\mathrm{N}$ were performed on an Elementar vario MICRO cube at the Elemental Analysis Centre of School of Science at the University of Tokyo. Thermogravimetric analysis and differential thermal analyses (TG-DTA) were performed on a Rigaku Thermo Plus TG 8120. Powder XRD patterns were recorded on a Rigaku SmartLab diffractometer $(\mathrm{CuK \alpha}, \lambda=1.5405 \AA, 45 \mathrm{kV}, 200 \mathrm{~mA})$. The XPS spectrum was measured on an Ulvac-Phi PHI5000 VersaProbe at the Advanced Characterisation Nanotechnology Platform of the University of Tokyo. XANES spectra were measured by a transmission method at the beam line BL11S2 at the Aichi Synchrotron Radiation Center. Each sample was mixed with boron nitride and formed into a pellet. The pellets were doubly wrapped in Al laminate packs and heat-sealed in an Ar-filled glovebox. Ag foil was used for energy calibrations.

Single-crystal X-ray crystallography: Diffraction measurements were made on a Rigaku VariMax Saturn 724 diffractometer with graphite monochromated Mo Ka radiation $(\lambda=0.71069 \AA, 50 \mathrm{kV}, 24 \mathrm{~mA})$ at $123 \mathrm{~K}$. The data were collected using CrystalClear and processed using CrysAlis ${ }^{\text {Pro }} .{ }^{3}$ Neutral scattering factors were obtained from the standard source. In the reduction of data, Lorentz and polarization corrections were made. The structural analyses were performed using WinGX. ${ }^{\mathrm{S} 4}$ All structures were solved by SHELXS-97 (direct methods) and refined by SHELXL-2018/3. ${ }^{\mathrm{S} 5}$ The metal atoms (Si, Ag and W) and oxygen atoms in the POM 
frameworks were refined anisotropically. The highly disordered TBA cations and solvent molecules were omitted by use of SQUEEZE program. ${ }^{\text {S6 }}$ CCDC-1949945, CCDC-1949946 and CCDC-1949947 contain the supplementary crystallographic data for Ag2, Ag6 and Ag27, respectively. These data can be obtained free of charge from The Cambridge Crystallographic Data Centre via www.ccdc.cam.ac.uk/data_request/cif.

Bond valence sum (BVS) calculation: The BVS values were calculated by the expression for the variation of the length $\mathrm{r}_{i j}$ of a bond between two atoms $i$ and $j$ in observed crystal with valence $\mathrm{V}_{i}$.

$$
V_{i}=\sum_{j} \exp \left(\frac{r_{0}^{\prime}-r_{i j}}{B}\right)
$$

where $\mathrm{B}$ is constant equal to $0.37 \AA, r_{0}{ }_{0}$ is bond valence parameter for a given atom pair. ${ }^{\mathrm{S} 7}$

Computational details: The geometries used in the calculations were obtained from the crystal structure determined in this study. We performed the calculations in acetone and the solvent effects were included using the polarizable continuum model (PCM). We employed the moderate-size basis set $\left(6-31 \mathrm{G}^{*}\right.$ for $\mathrm{O}$ and $\mathrm{Si}$, and LANL2DZ ECP for $\mathrm{W}$ and Ag) because the size of the target molecule is large. All calculations were performed with Gaussian 16.

Synthesis and characterisation of $\mathrm{TBA}_{7} \mathrm{H}_{2}\left[\mathrm{Ag}_{2}\left(\mathrm{SiW}_{9} \mathrm{O}_{31}\right)_{2}\left(\mathrm{CH}_{3} \mathrm{COO}\right)_{3}\right] \cdot \mathrm{H}_{2} \mathrm{O} \cdot \mathrm{CCl}_{4}(\mathrm{Ag} 2)$ : To silver acetate (5.3 mg, $30.8 \mu \mathrm{mol})$ in a mixture of acetone $(2 \mathrm{~mL}), \mathrm{TBA}_{4}\left[\mathrm{SiW}_{9} \mathrm{O}_{28}\left(\mathrm{OCH}_{3}\right)_{6}\right](101 \mathrm{mg}, 30.8 \mu \mathrm{mol})$ was added. The resulting solution was stirred for $5 \mathrm{~h}$ at room temperature. Colorless single crystals of Ag2 suitable for X-ray crystallography were obtained from a mixture of the synthetic solution and carbon tetrachloride (52.6 mg, 48\% yield). IR (KBr pellet): 3447, 2961, 2935, 2874, 1637, 1484, 1381, 1153, 1108, 1054, 999, 953, 906, 768, 667, 557, $457 \mathrm{~cm}^{-1} .{ }^{29} \mathrm{Si} \mathrm{NMR}\left(99.37 \mathrm{MHz}\right.$, acetone- $d_{6}$ solution containing $1 \mathrm{M}$ ethylene carbonate, 298K): $\delta=-83.6 \mathrm{ppm}$. UV-Vis (acetone solution containing $1 \mathrm{M}$ ethylene carbonate): $\lambda(\varepsilon) 350 \mathrm{~nm}(3.92 \times$ $\left.10^{3} \mathrm{M}^{-1} \mathrm{~cm}^{-1}\right)$. ESI-mass (positive mode, acetone): $\mathrm{m} / z 3437.074\left[\mathrm{TBA}_{9} \mathrm{HAg}_{2}\left(\mathrm{SiW}_{9} \mathrm{O}_{31}\right)_{2}\left(\mathrm{CH}_{3} \mathrm{COO}\right)_{2}\right]^{2+}($ calcd. 3437.085), $3467.094\left[\mathrm{TBA}_{9} \mathrm{H}_{2} \mathrm{Ag}_{2}\left(\mathrm{SiW}_{9} \mathrm{O}_{31}\right)_{2}\left(\mathrm{CH}_{3} \mathrm{COO}\right)_{3}\right]^{2+}$ (calcd. 3467.096). Elemental analysis: calcd (\%) for $\mathrm{TBA}_{7} \mathrm{H}_{2}\left[\mathrm{Ag}_{2}\left(\mathrm{SiW}_{9} \mathrm{O}_{31}\right)_{2}\left(\mathrm{CH}_{3} \mathrm{COO}\right)_{3}\right] \cdot \mathrm{H}_{2} \mathrm{O} \cdot \mathrm{CCl}_{4}, \mathrm{C} 21.59, \mathrm{H}$ 4.03, N 1.48, Si 0.85, Ag 3.26, W 49.98; Found C 21.30, H 4.07, N 1.50, Si 0.80, Ag 3.19, W 49.98. 


\section{Synthesis and characterisation of $\mathrm{TBA}_{16}\left(\mathrm{Me}_{2} \mathrm{NH}_{2}\right)_{8} \mathrm{H}_{5} \mathrm{Ag}_{2}\left[\mathrm{Ag}_{27}\left(\mathrm{Si}_{2} \mathrm{~W}_{18} \mathrm{O}_{66}\right)_{3}\right] \cdot 12 \mathrm{C}_{3} \mathrm{H}_{4} \mathrm{O}_{3} \cdot 34 \mathrm{H}_{2} \mathrm{O}(\mathrm{Ag27})$ :}

To Ag2 $(15.4 \mu \mathrm{mol})$ in a mixture of acetone $(2 \mathrm{~mL})$ and water $(10 \mu \mathrm{L}), N, N$-dimethylformamide $(1 \mathrm{~mL})$ and silver benzoate $(134 \mathrm{mg}, 624 \mu \mathrm{mol})$ were added. The resulting solution was stirred for $48 \mathrm{~h}$. During the reaction, the colorless solution gradually turned dark brown. Diethyl ether $(30 \mathrm{~mL})$ was added and dark brown precipitates of Ag27 were obtained. Ag27 could also be synthesised by the following procedure: To silver benzoate $(35.5 \mathrm{mg}, 154 \mu \mathrm{mol})$ in a mixture of acetone $(10 \mathrm{~mL})$ and water $(50 \mu \mathrm{L}), \mathrm{TBA}_{4}\left[\mathrm{SiW}_{9} \mathrm{O}_{28}\left(\mathrm{OCH}_{3}\right)_{6}\right]$ (506 mg, $154 \mu \mathrm{mol}$ ) was added. The resulting solution was stirred for $3 \mathrm{~h}$, and then $N, N$-dimethylformamide $(5 \mathrm{~mL})$ and silver benzoate $(134 \mathrm{mg}, 624 \mu \mathrm{mol})$ were added. The subsequent solution was then stirred for an additional $48 \mathrm{~h}$ at room temperature. Diethyl ether $(300 \mathrm{~mL})$ was added, and dark brown precipitates of Ag27 were obtained. The precipitates were filtered off and then recrystallized from an acetone solution containing ethylene carbonate (2.9 M) and ethyl acetate to give crystals of Ag27 (236 mg, 40\% yield). The single crystals suitable for X-ray crystallography were obtained by further recrystallization from an acetone solution containing ethylene carbonate (1 M) and diethyl ether. IR (KBr pellet): 3448, 2960, 2937, 2874, 1800, 1773, $1629,1486,1466,1384,1153,1072,993,931$ (sh), 871, 737, 664, 553, 520 (sh) $\mathrm{cm}^{-1} .{ }^{29} \mathrm{Si} \mathrm{NMR}(99.37 \mathrm{MHz}$ acetone- $d_{6}$ solution containing $1 \mathrm{M}$ ethylene carbonate, $\left.298 \mathrm{~K}, \mathrm{Si}\left(\mathrm{CH}_{3}\right)_{4}\right) \delta=-79.2 \mathrm{ppm}$. UV-Vis (acetone solution containing $1 \mathrm{M}$ ethylene carbonate): $\lambda(\varepsilon) 600 \mathrm{~nm}\left(9.05 \times 10^{3} \mathrm{M}^{-1} \mathrm{~cm}^{-1}\right), 530 \mathrm{~nm}\left(7.09 \times 10^{3}\right.$ $\left.\mathrm{M}^{-1} \mathrm{~cm}^{-1}\right), 435 \mathrm{~nm}\left(6.65 \times 10^{4} \mathrm{M}^{-1} \mathrm{~cm}^{-1}\right), 530 \mathrm{~nm}\left(7.09 \times 10^{3} \mathrm{M}^{-1} \mathrm{~cm}^{-1}\right), 350 \mathrm{~nm}\left(7.60 \times 10^{4} \mathrm{M}^{-1} \mathrm{~cm}^{-1}\right)$.

ESI-mass (positive mode, acetone): $\mathrm{m} / \mathrm{z} 5205.968$ assignable to $\left[\mathrm{TBA}_{16}\left(\mathrm{Me}_{2} \mathrm{NH}_{2}\right)_{6} \mathrm{H}_{11} \mathrm{Ag}_{29}\left(\mathrm{Si}_{2} \mathrm{~W}_{18} \mathrm{O}_{66}\right)_{3}\left(\mathrm{C}_{3} \mathrm{H}_{4} \mathrm{O}_{3}\right)_{3}\right]^{4+}$ (theoretical $\mathrm{m} / \mathrm{z}$ : 5205.888). Elemental analysis: calcd (\%) for $\mathrm{TBA}_{16}\left(\mathrm{Me}_{2} \mathrm{NH}_{2}\right)_{8} \mathrm{H}_{5} \mathrm{Ag}_{2}\left[\mathrm{Ag}_{27}\left(\mathrm{Si}_{2} \mathrm{~W}_{18} \mathrm{O}_{66}\right)_{3}\right] \cdot 12 \mathrm{C}_{3} \mathrm{H}_{4} \mathrm{O}_{3} \cdot 34 \mathrm{H}_{2} \mathrm{O}, \mathrm{C}$ 16.58, H 3.44, N 1.51, Si 0.76, $\mathrm{Ag} 14.02$, W 44.49; found, C 16.35, H 3.19, N 1.51, Si 0.74, Ag 13.90, W 44.66. 
Table S1. Reported silver nanoclusters using organic ligands with X-ray crystallographic analyses.

\begin{tabular}{|c|c|c|}
\hline Compound & & References \\
\hline $\mathrm{Ag}_{14}\left(\mathrm{SC}_{6} \mathrm{H}_{3} \mathrm{~F}_{2}\right)_{12}\left(\mathrm{PPh}_{3}\right)_{8}$ & & [S8] \\
\hline$\left[\mathrm{Ag}_{15}\left\{\mathrm{~N}\left(\mathrm{CH}_{2} \mathrm{PPh}_{2}\right)_{3}\right\}_{4} \mathrm{Cl}_{4}\right]\left(\mathrm{NO}_{3}\right)_{3}$ & & [S9] \\
\hline $\mathrm{Ag}_{16}\left\{\mathrm{Ph}_{2} \mathrm{P}\left(\mathrm{CH}_{2}\right)_{2} \mathrm{PPh}_{2}\right\}_{4}\left(\mathrm{SC}_{6} \mathrm{H}_{3} \mathrm{~F}_{2}\right)_{14}$ & & {$[\mathrm{~S} 10]$} \\
\hline$\left[\mathrm{Ag}_{19}\left\{\left(\mathrm{Ph}_{2} \mathrm{P}\right)_{2} \mathrm{CH}_{2}\right\}_{3}\left(\mathrm{PhC}_{2}\right)_{14}\right]\left(\mathrm{SbF}_{6}\right)_{3}$ & & {$[\mathrm{~S} 11]$} \\
\hline $\mathrm{Ag}_{20}\left\{\mathrm{~S}_{2} \mathrm{P}\left(\mathrm{O}^{i} \mathrm{Pr}\right)_{2}\right\}_{12}$ & & {$[\mathrm{~S} 12]$} \\
\hline $\mathrm{Ag}_{20}\left\{\mathrm{Se}_{2} \mathrm{P}\left(\mathrm{O}^{i} \mathrm{Pr}\right)_{2}\right\}_{12}$ & & {$[\mathrm{~S} 13]$} \\
\hline$\left[\mathrm{Ag}_{21}\left\{\mathrm{~S}_{2} \mathrm{P}\left(\mathrm{O}^{i} \mathrm{Pr}\right)_{2}\right\}\right]_{12}^{+}$ & & {$[\mathrm{S} 14]$} \\
\hline$\left[\mathrm{Ag}_{21}\left\{\mathrm{Se}_{2} \mathrm{P}(\mathrm{OEt})_{2}\right\}_{12}\right]^{+}$ & & {$[\mathrm{S} 13]$} \\
\hline $\mathrm{Ag}_{23}\left(\mathrm{PPh}_{3}\right)_{8}\left(\mathrm{SC}_{2} \mathrm{H}_{4} \mathrm{Ph}\right)_{18}$ & & {$[\mathrm{~S} 15]$} \\
\hline$\left[\mathrm{Ag}_{25}\left(\mathrm{SPhMe}_{2}\right)_{18}\right]^{-}$ & & {$[\mathrm{S} 16]$} \\
\hline$\left[\mathrm{Ag}_{25}\left\{\mathrm{Ph}_{2} \mathrm{P}\left(\mathrm{CH}_{2}\right)_{5} \mathrm{PPh}_{2}\right\}_{3}\left(\mathrm{MeOPhC}_{2}\right)_{20}\right]\left(\mathrm{SbF}_{6}\right)_{3}$ & & {$[\mathrm{~S} 11]$} \\
\hline $\mathrm{Ag}_{29}(1,3 \text {-benzendithiol })_{12}\left(\mathrm{Ph}_{3} \mathrm{P}\right)_{4}$ & & {$[\mathrm{~S} 17]$} \\
\hline$\left[\mathrm{Ag}_{32}\left\{\mathrm{Ph}_{2} \mathrm{P}\left(\mathrm{CH}_{2}\right)_{2} \mathrm{PPh}_{2}\right\}_{5}\left(\mathrm{SC}_{6} \mathrm{H}_{3} \mathrm{~F}_{2}\right)_{24}\right]^{2-}$ & & {$[\mathrm{S} 11]$} \\
\hline$\left[\mathrm{Ag}_{34} \mathrm{~S}_{2}\left(\mathrm{SCH}_{2} \mathrm{Ph}\right)_{18}\left(\mathrm{CF}_{3} \mathrm{COO}\right)_{9}(\mathrm{DMF})_{6}\right]$ & & {$[\mathrm{S} 17]$} \\
\hline$\left[\mathrm{Ag}_{34}\left(\mathrm{CO}_{3}\right)_{12} \mathrm{Cl}_{4}\left\{\left(\mathrm{Ph}_{2} \mathrm{P}\right)_{2} \mathrm{CH}_{2}\right\}_{12}\right]$ & & {$[\mathrm{S} 18]$} \\
\hline$\left[\mathrm{Ag}_{35}\left(\mathrm{H}_{2} \mathrm{~L}\right)_{2}(\mathrm{~L})\left(\mathrm{C}_{2}{ }^{t} \mathrm{Bu}\right)_{16}\right]\left(\mathrm{SbF}_{6}\right)_{3} \quad(\mathrm{~L}=$ Thiacalixarene $)$ & & {$[\mathrm{S} 19]$} \\
\hline$\left[\mathrm{Ag}_{40}\left(\mathrm{SPhMe}_{2}\right)_{24}\left(\mathrm{PPh}_{3}\right)_{8}\right]$ & & {$[\mathrm{S} 20]$} \\
\hline$\left[\mathrm{Ag}_{40}\left(\mathrm{SPhMe}_{2}\right)_{24}\left(\mathrm{PPh}_{3}\right)_{8} \mathrm{H}_{12}\right]^{2+}$ & & {$[\mathrm{S} 21]$} \\
\hline$\left[\mathrm{Ag}_{43}\left(\mathrm{SPhMe}_{2}\right)_{25}\left(\mathrm{PPh}_{3}\right)_{4}\right]$ & & {$[\mathrm{S} 20]$} \\
\hline $\mathrm{Ag}_{44}(\mathrm{SR})_{30}\left(\mathrm{R}=\mathrm{PhCF}_{3}, \mathrm{SPhF}_{2}, \mathrm{SPhF}\right)$ & & {$[\mathrm{S} 22]$} \\
\hline$\left[\mathrm{Ag}_{44}(\mathrm{SPhCOOH})_{30}\right]^{4-}$ & & {$[\mathrm{S} 23]$} \\
\hline$\left[\mathrm{Ag}_{46}\left(\mathrm{SPhMe}_{2}\right)_{24}\left(\mathrm{PPh}_{3}\right)_{8}\right]^{2+}$ & & {$[\mathrm{S} 20]$} \\
\hline$\left[\mathrm{Ag}_{48}\left(\mathrm{C}_{2}{ }^{t} \mathrm{Bu}\right)_{20}\left(\mathrm{CrO}_{4}\right)_{7}\right]$ & & {$[\mathrm{S} 24]$} \\
\hline $\mathrm{Ag}_{50}\left\{\left(\mathrm{Ph}_{2} \mathrm{P}\right)_{2} \mathrm{CH}_{2}\right\}_{6}\left(\mathrm{SCH}_{2} \mathrm{Ph}^{t} \mathrm{Bu}\right)_{30}$ & & {$[\mathrm{~S} 25]$} \\
\hline$\left[\mathrm{Ag}_{51}\left({ }^{t} \mathrm{BuC}_{2}\right)_{32}\right]^{3+}$ & & {$[\mathrm{S} 26]$} \\
\hline$\left[\mathrm{Ag}_{62} \mathrm{~S}_{12}\left(\mathrm{~S}{ }^{t} \mathrm{Bu}\right)_{32}\right]^{2+}$ & & {$[\mathrm{S} 27]$} \\
\hline$\left[\mathrm{Ag}_{67}\left(\mathrm{SPhMe}_{2}\right)_{32}\left(\mathrm{PPh}_{3}\right)_{8}\right]^{3+}$ & & {$[\mathrm{S} 28]$} \\
\hline$\left[\mathrm{Ag}_{74}\left(\mathrm{C}_{2} \mathrm{Ph}\right)_{44}\right]\left(\mathrm{NO}_{3}\right)_{2}$ & & [S29] \\
\hline $\mathrm{Ag}_{78}\left\{\mathrm{Ph}_{2} \mathrm{P}\left(\mathrm{CH}_{2}\right)_{3} \mathrm{PPh}_{2}\right\}_{6}(\mathrm{SPhCF} 3)_{42}$ & & {$[\mathrm{~S} 30]$} \\
\hline$\left[\mathrm{Ag}_{141} \mathrm{X}_{12}\left(\mathrm{SC}_{10} \mathrm{H}_{15}\right)_{40}\right]^{3+}(\mathrm{X}=\mathrm{Cl}, \mathrm{Br})$ & & {$[\mathrm{S} 31]$} \\
\hline $\mathrm{Ag}_{146} \mathrm{Br}_{2}\left(\mathrm{SPh}^{i} \mathrm{Pr}\right)_{80}$ and $\mathrm{Ag}_{150} \mathrm{Br}_{2}\left(\mathrm{SPh}^{i} \mathrm{Pr}\right)_{84}$ & (cocrystal) & {$[\mathrm{S} 32]$} \\
\hline$\left[\mathrm{Ag}_{206}\left(\mathrm{SC}_{6} \mathrm{H}_{11}\right)_{68} \mathrm{~F}_{2} \mathrm{Cl}_{2}\right]^{4+}$ & & {$[\mathrm{S} 33]$} \\
\hline $\mathrm{Ag}_{210}\left({ }^{i} \mathrm{PrPhS}\right)_{71}\left(\mathrm{PPh}_{3}\right)_{5} \mathrm{Cl}$ and $\mathrm{Ag}_{211}\left({ }^{i} \mathrm{PrPhS}\right)_{71}\left(\mathrm{PPh}_{3}\right)_{6} \mathrm{Cl}$ & (cocrystal) & {$[\mathrm{S} 34]$} \\
\hline $\mathrm{Ag}_{374}\left(\mathrm{SPh}^{t} \mathrm{Bu}\right)_{113} \mathrm{Br}_{2} \mathrm{Cl}_{2}$ & & {$[\mathrm{~S} 35]$} \\
\hline
\end{tabular}


Table S2. Reported silver nanoclusters using oxometalates as inorganic ligands with X-ray crystallographic analyses.

\begin{tabular}{llc}
\hline Compound & $\mathrm{Ag}$ core & References \\
\hline$\left(n-\mathrm{Bu}_{4} \mathrm{~N}\right)_{8}\left[\mathrm{Ag}_{6}\left(\mathrm{H}_{2} \mathrm{SiW}_{10} \mathrm{O}_{36}\right)_{2}\right] \cdot 5 \mathrm{H}_{2} \mathrm{O}$ & $\mathrm{Ag}_{6}$ & {$[\mathrm{~S} 36]$} \\
{$\left[\mathrm{Ag}_{6} @\left(\mathrm{MoO}_{4}\right)_{7} @ \mathrm{Ag}_{56}\left(\mathrm{MoO}_{4}\right)_{2}\left({ }^{i} \mathrm{PrS}\right)_{28}\left(\mathrm{NO}_{3}\right)_{14}\right]$} & $\mathrm{Ag}_{6}$ & {$[\mathrm{~S} 37]$} \\
{$\left[\mathrm{Ag}\left(\mathrm{CH}_{3} \mathrm{CN}\right)_{2}\left[\mathrm{Ag}_{6} \mathrm{Ti}_{16}\left(\mu_{3}-\mathrm{O}\right)_{18}\left(\mu_{2}-\mathrm{O}\right)_{4}\left(\mathrm{PhCO}_{2}\right)_{26}\left(\mathrm{CH}_{3} \mathrm{CN}\right)_{2}\right]\right.$} & $\mathrm{Ag}_{6}$ & {$[\mathrm{~S} 38]$} \\
{$\left[\mathrm{Ag}_{10} @\left(\mathrm{Mo}_{7} \mathrm{O}_{26}\right)_{2} @ \mathrm{Ag}_{70}\left(\mathrm{MoO}_{4}\right)_{2}\left(\mathrm{SC}_{6} \mathrm{H}_{11}\right)_{36}\left(\mathrm{CF}_{3} \mathrm{SO}_{3}\right)_{16}(\mathrm{DMF})_{6}\right]$} & $\mathrm{Ag}_{10}$ & {$[\mathrm{~S} 39]$} \\
{$\left[\mathrm{Ag}_{10} @\left(\mathrm{MoO}_{4}\right)_{7} @ \mathrm{Ag}_{60}\left({ }^{t} \mathrm{BuC}_{6} \mathrm{H}_{4} \mathrm{~S}\right)_{33}(\mathrm{MeOPhCO})_{18}(\mathrm{DMF})\left(\mathrm{H}_{2} \mathrm{O}\right)_{2}\right] \cdot \mathrm{DMF} \cdot \mathrm{H}_{2} \mathrm{O}$} & $\mathrm{Ag}_{10}$ & {$[\mathrm{~S} 40]$} \\
\hline
\end{tabular}


Table S3. X-ray crystallographic data for Ag2, Ag27, and Ag6.

\begin{tabular}{llll}
\hline & Ag2 & Ag27 & Ag6 \\
\hline Formula & $\mathrm{C}_{120} \mathrm{H}_{261} \mathrm{Ag}_{2} \mathrm{Cl}_{8} \mathrm{~N}_{7} \mathrm{O}_{68} \mathrm{Si}_{2} \mathrm{~W}_{18}$ & $\mathrm{C}_{146} \mathrm{H}_{320} \mathrm{Ag}_{29} \mathrm{~N}_{13} \mathrm{O}_{236} \mathrm{Si}_{6} \mathrm{~W}_{54}$ & $\mathrm{C}_{120} \mathrm{H}_{268} \mathrm{Ag}_{6} \mathrm{Cl}_{8} \mathrm{~N}_{7} \mathrm{O}_{68} \mathrm{Si}_{2} \mathrm{~W}_{18}$ \\
$\mathrm{FW}\left(\mathrm{g} \mathrm{mol}{ }^{-1}\right)$ & 6755.16 & 19258.80 & 7193.70 \\
Crystal system & Monoclinic & Triclinic & Monoclinic \\
Space group & $P 2 / c$ & $P-1$ & $C 2 / c$ \\
$a(\AA)$ & $19.2160(5)$ & $27.7691(2)$ & $40.0068(6)$ \\
$b(\AA)$ & $14.5222(3)$ & $33.0762(2)$ & $16.6856(2)$ \\
$c(\AA)$ & $38.5485(9)$ & $35.0235(2)$ & $29.7859(5)$ \\
$\alpha(\mathrm{deg})$ & 90 & $83.5033(5)$ & 90 \\
$\beta(\mathrm{deg})$ & $102.952(2)$ & $68.9814(6)$ & $100.2067(16)$ \\
$\gamma(\mathrm{deg})$ & 90 & $69.7620(6)$ & 90 \\
$V\left(\AA^{3}\right)$ & $10483.6(4)$ & $28172.5(3)$ & $19568.5(5)$ \\
$Z$ & 2 & 2 & 4 \\
Temp $(\mathrm{K})$ & $123(2)$ & $123(2)$ & $123(2)$ \\
$\rho_{\text {calcd }}(\mathrm{g} \mathrm{cm}$ & 2.140 & 2.270 & 2.442 \\
$\mathrm{GOF}$ & 1.057 & 1.012 & 1.034 \\
$R_{1}[I>2 \sigma(I)]$ & 0.0894 & 0.0622 & 0.0446 \\
$w R_{2}$ & 0.2241 & 0.1734 & 0.1030 \\
\hline
\end{tabular}

Table S4. BVS values for silicon and tungsten atoms of Ag2.

\begin{tabular}{llllll}
\hline Si1 & 4.02 & Ag1 & 0.97 & & \\
W1 & 6.11 & W4 & 6.39 & W7 & 6.17 \\
W2 & 6.29 & W5 & 6.09 & W8 & 6.13 \\
W3 & 6.06 & W6 & 6.32 & W9 & 6.11 \\
\hline
\end{tabular}


Table S5. Distances ( $\AA$ ) between silver atoms of Ag27.

\begin{tabular}{|c|c|c|c|c|c|c|c|c|c|c|c|}
\hline Ag1 & $\ldots$ & Ag2 & 2.95 & Ag7 & $\ldots$ & Ag8 & 2.96 & Ag13 & $\ldots$ & Ag14 & 2.96 \\
\hline $\mathrm{Ag} 1$ & $\ldots$ & $\mathrm{Ag} 3$ & 2.75 & $\mathrm{Ag} 7$ & $\ldots$ & $\mathrm{Ag} 9$ & 2.74 & Ag13 & $\ldots$ & Ag15 & 2.75 \\
\hline $\operatorname{Ag} 1$ & $\ldots$ & $\mathrm{Ag} 4$ & 2.95 & $\mathrm{Ag} 7$ & $\ldots$ & $\operatorname{Ag} 10$ & 2.97 & $\operatorname{Ag} 13$ & $\ldots$ & $\operatorname{Ag} 16$ & 2.94 \\
\hline Ag1 & $\ldots$ & Ag5 & 2.75 & $\mathrm{Ag} 7$ & $\ldots$ & Ag11 & 2.75 & Ag13 & $\ldots$ & Ag17 & 2.75 \\
\hline Ag2 & $\ldots$ & Ag3 & 2.73 & Ag8 & $\ldots$ & $\mathrm{Ag} 9$ & 2.73 & $\operatorname{Ag} 14$ & $\ldots$ & $\operatorname{Ag} 15$ & 2.73 \\
\hline $\mathrm{Ag} 2$ & $\ldots$ & Ag5 & 2.72 & Ag8 & $\ldots$ & Ag11 & 2.71 & Ag14 & $\ldots$ & Ag17 & 2.73 \\
\hline Ag2 & $\ldots$ & Ag6 & 2.98 & Ag8 & $\ldots$ & $\operatorname{Ag} 12$ & 3.02 & Ag14 & $\ldots$ & $\operatorname{Ag} 18$ & 2.98 \\
\hline Ag3 & $\ldots$ & $\mathrm{Ag} 4$ & 2.72 & $\mathrm{Ag} 9$ & $\ldots$ & Ag10 & 2.72 & Ag15 & $\ldots$ & Ag16 & 2.72 \\
\hline Ag3 & $\ldots$ & Ag6 & 2.77 & $\mathrm{Ag} 9$ & $\ldots$ & Ag12 & 2.75 & Ag15 & $\ldots$ & Ag18 & 2.74 \\
\hline $\mathrm{Ag} 4$ & $\ldots$ & Ag5 & 2.72 & Ag10 & $\ldots$ & Ag11 & 2.73 & Ag16 & $\ldots$ & Ag17 & 2.73 \\
\hline $\mathrm{Ag} 4$ & $\ldots$ & Ag6 & 2.98 & $\mathrm{Ag} 10$ & $\ldots$ & $\operatorname{Ag} 12$ & 3.00 & Ag16 & $\ldots$ & $\operatorname{Ag} 18$ & 2.98 \\
\hline Ag5 & $\ldots$ & Ag6 & 2.76 & $\mathrm{Ag} 11$ & $\ldots$ & Ag12 & 2.74 & Ag17 & $\ldots$ & $\operatorname{Ag} 18$ & 2.76 \\
\hline Ag6 & $\ldots$ & Ag12 & 2.81 & Ag12 & $\ldots$ & Ag18 & 2.79 & Ag18 & $\ldots$ & Ag6 & 2.81 \\
\hline Ag6 & $\ldots$ & Ag19 & 2.87 & Ag12 & $\ldots$ & $\operatorname{Ag} 20$ & 2.89 & Ag18 & $\ldots$ & $\operatorname{Ag} 21$ & 2.88 \\
\hline Ag6 & $\ldots$ & Ag22 & 2.82 & $\mathrm{Ag} 12$ & $\ldots$ & Ag23 & 2.81 & Ag18 & $\ldots$ & $\operatorname{Ag} 24$ & 2.82 \\
\hline Ag21 & $\ldots$ & Ag6 & 2.82 & $\operatorname{Ag} 19$ & $\ldots$ & Ag12 & 2.81 & Ag20 & $\ldots$ & $\operatorname{Ag} 18$ & 2.80 \\
\hline $\operatorname{Ag} 24$ & $\ldots$ & Ag6 & 2.86 & $\mathrm{Ag} 22$ & $\ldots$ & Ag12 & 2.88 & Ag23 & $\ldots$ & Ag18 & 2.88 \\
\hline $\operatorname{Ag} 21$ & $\ldots$ & Ag19 & 2.76 & Ag19 & $\ldots$ & Ag20 & 2.75 & Ag20 & $\ldots$ & $\operatorname{Ag} 21$ & 2.79 \\
\hline $\mathrm{Ag} 24$ & $\ldots$ & Ag22 & 2.76 & $\mathrm{Ag} 22$ & $\ldots$ & $\operatorname{Ag} 23$ & 2.76 & Ag23 & $\ldots$ & $\mathrm{Ag} 24$ & 2.74 \\
\hline Ag3 & $\ldots$ & Ag25 & 2.81 & $\mathrm{Ag} 9$ & $\ldots$ & Ag26 & 2.84 & Ag15 & $\ldots$ & Ag27 & 2.87 \\
\hline Ag5 & $\ldots$ & Ag27 & 2.81 & $\mathrm{Ag} 11$ & $\ldots$ & Ag25 & 2.87 & Ag17 & $\ldots$ & $\mathrm{Ag} 26$ & 2.82 \\
\hline
\end{tabular}


Table S6. BVS values for silicon and tungsten atoms of Ag27.

\begin{tabular}{cccccc}
\hline Si1 & 3.93 & Si3 & 3.94 & Si5 & 3.91 \\
Si2 & 3.95 & Si4 & 3.89 & Si6 & 3.89 \\
Ag31 & 1.02 & Ag32 & 0.97 & & \\
W1 & 6.19 & W19 & 6.01 & W37 & 6.04 \\
W2 & 6.20 & W20 & 6.31 & W38 & 6.19 \\
W3 & 6.24 & W21 & 6.08 & W39 & 6.31 \\
W4 & 6.04 & W22 & 6.25 & W40 & 6.06 \\
W5 & 5.92 & W23 & 6.11 & W41 & 6.09 \\
W6 & 6.25 & W24 & 6.09 & W42 & 6.21 \\
W7 & 6.10 & W25 & 6.27 & W43 & 6.15 \\
W8 & 6.14 & W26 & 6.07 & W44 & 6.06 \\
W9 & 6.18 & W27 & 6.41 & W45 & 6.10 \\
W10 & 6.14 & W28 & 6.01 & W47 & 6.24 \\
W11 & 6.10 & W29 & 6.19 & W48 & 6.08 \\
W12 & 6.13 & W30 & 6.36 & W49 & 6.24 \\
W13 & 6.05 & W31 & 6.02 & W50 & 6.18 \\
W14 & 6.12 & W32 & 6.22 & W51 & 5.96 \\
W15 & 6.15 & W33 & 6.03 & W52 & 6.11 \\
W16 & 6.10 & W34 & 6.15 & W53 & 6.11 \\
W17 & 6.24 & W35 & 6.31 & 6.20 \\
W18 & 6.17 & W36 & 6.07 & &
\end{tabular}

Table S7. BVS values for silicon and tungsten atoms of Ag6.

\begin{tabular}{llllll}
\hline Si1 & 3.91 & & & & \\
W1 & 6.16 & W4 & 6.11 & W7 & 6.10 \\
W2 & 6.18 & W5 & 6.10 & W8 & 6.17 \\
W3 & 6.20 & W6 & 6.09 & W9 & 6.11 \\
\hline
\end{tabular}

Table S8. Distances $(\AA)$ between silver atoms of Ag6.

\begin{tabular}{llllllll}
\hline $\mathrm{Ag} 1$ & $\cdots$ & $\mathrm{Ag} 2$ & 2.84 & $\mathrm{Ag} 2$ & $\cdots$ & $\mathrm{Ag} 3 *$ & 2.74 \\
$\mathrm{Ag} 1$ & $\cdots$ & $\mathrm{Ag} 3$ & 2.80 & $\mathrm{Ag} 2$ & $\cdots$ & $\mathrm{Ag} 4$ & 2.85 \\
$\mathrm{Ag} 2$ & $\cdots$ & $\mathrm{Ag} 3$ & 2.69 & $\mathrm{Ag} 3$ & $\cdots$ & $\mathrm{Ag} 4$ & 2.72 \\
\hline
\end{tabular}




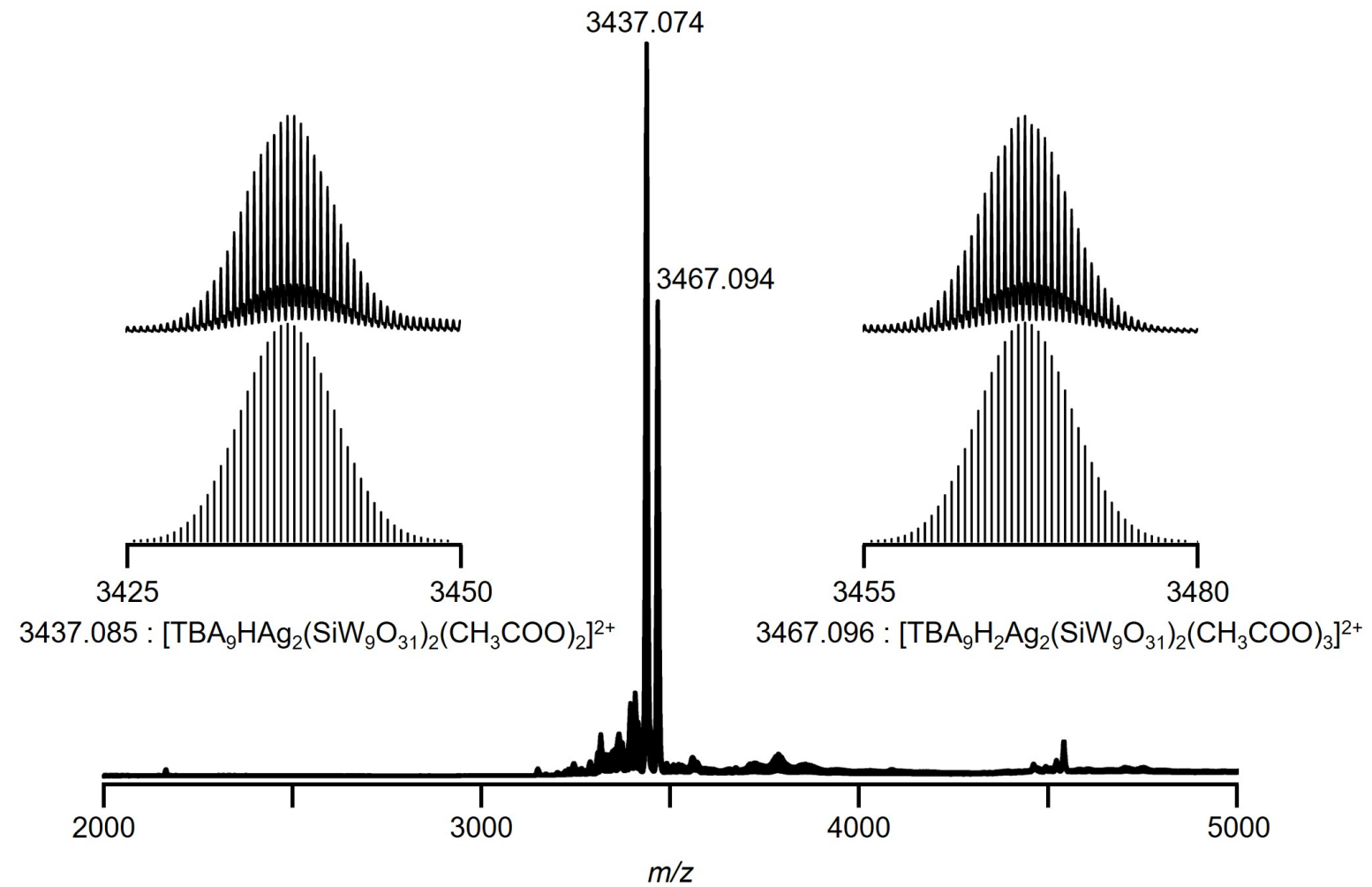

Figure S1. ESI-mass spectrum of Ag2 in acetone. Insets: spectra in the range of $m / z 3425-3450$ and 34553580 , and simulated patterns for $\left[\mathrm{TBA}_{9} \mathrm{HAg}_{2}\left(\mathrm{SiW}_{9} \mathrm{O}_{33}\right)_{2}\left(\mathrm{CH}_{3} \mathrm{COO}\right)_{2}\right]^{2+} \quad(m / z \quad 3437.085)$ and $\left[\mathrm{TBA}_{9} \mathrm{H}_{2} \mathrm{Ag}_{2}\left(\mathrm{SiW}_{9} \mathrm{O}_{33}\right)_{2}\left(\mathrm{CH}_{3} \mathrm{COO}\right)_{3}\right]^{2+}(\mathrm{m} / z$ 3467.096).

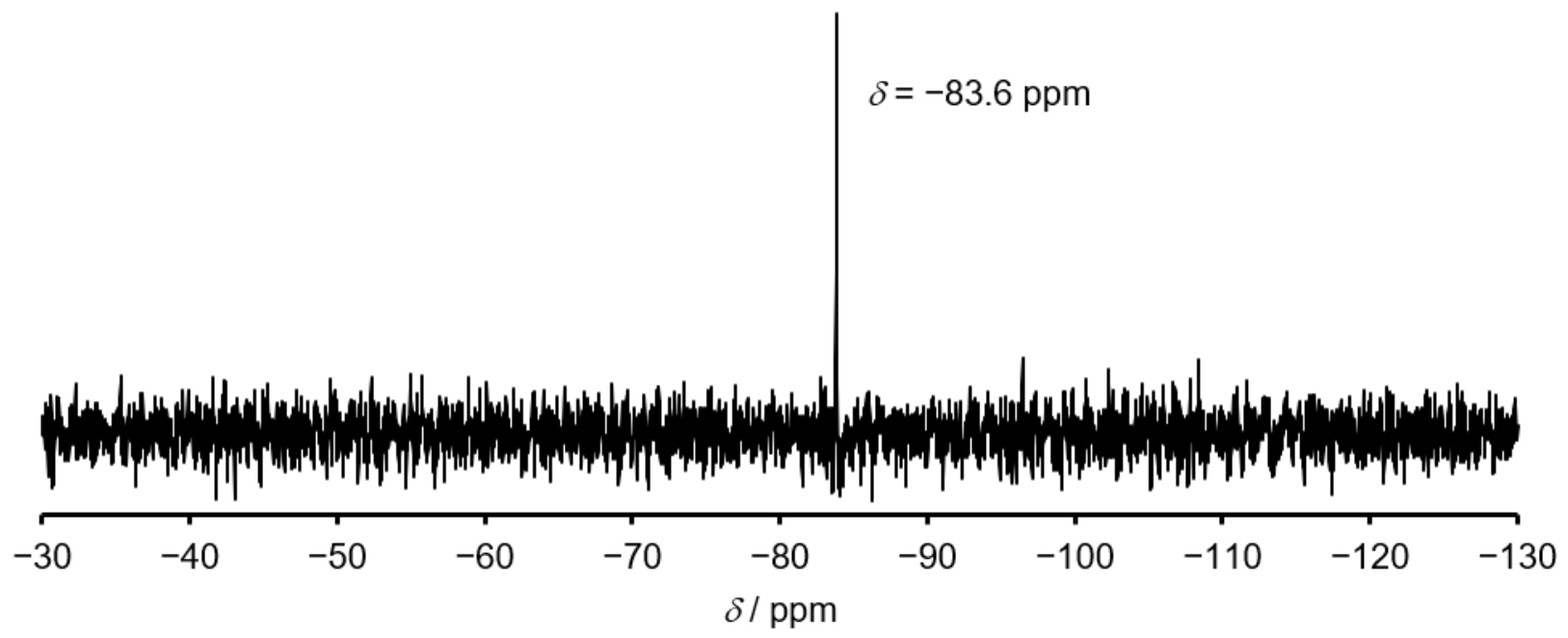

Figure S2. ${ }^{29} \mathrm{Si}$ NMR spectrum of Ag2 in an acetone- $d_{6}$ solution solution containing 1 M ethylene carbonate. 


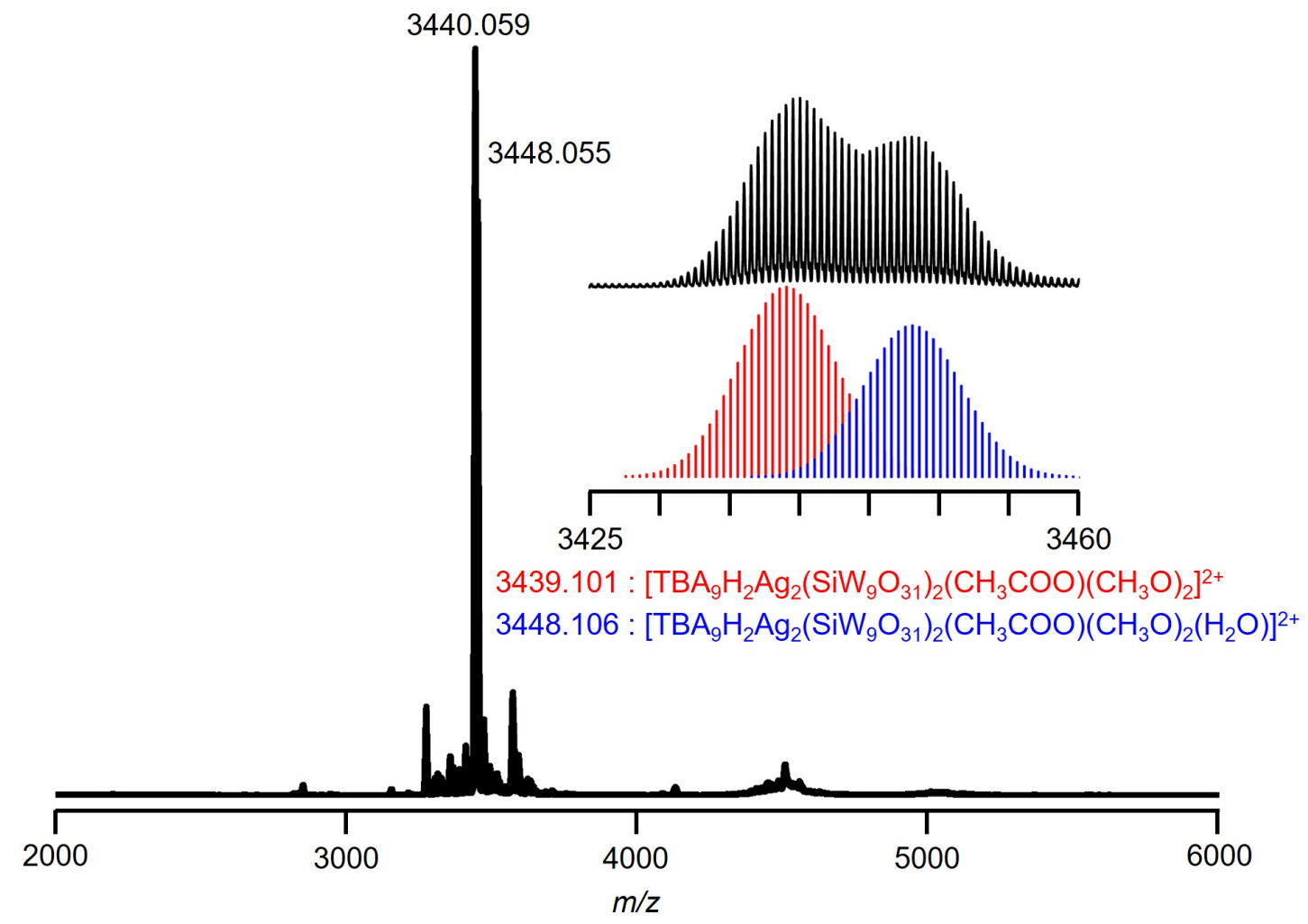

Figure S3. ESI-mass spectrum of the reaction solution of Ag2 and silver acetate (4 equiv with respect to Ag2) in acetone. 
(a)

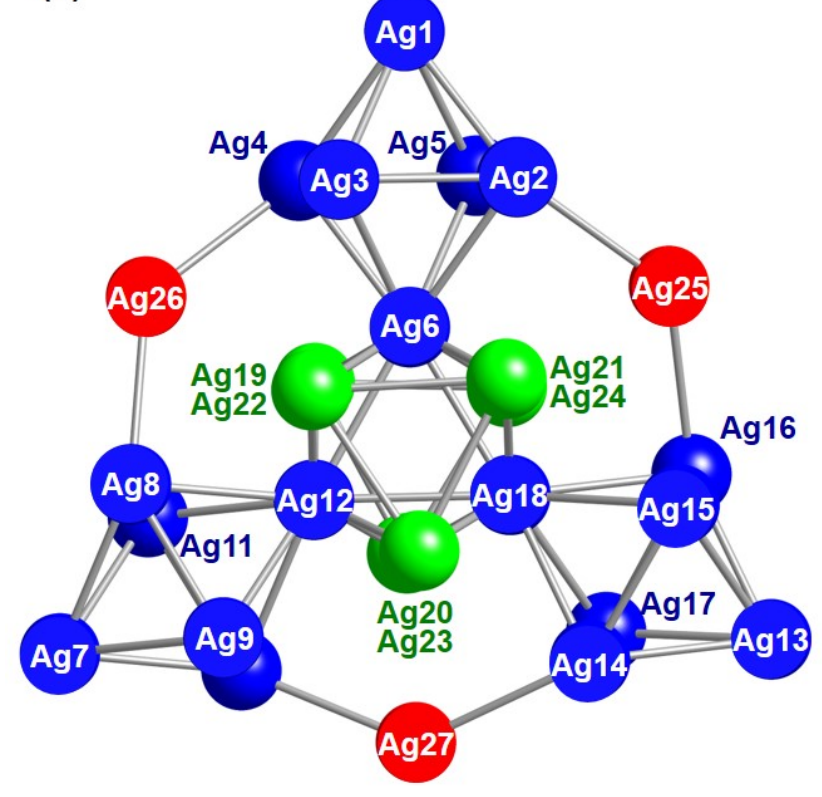

(b)

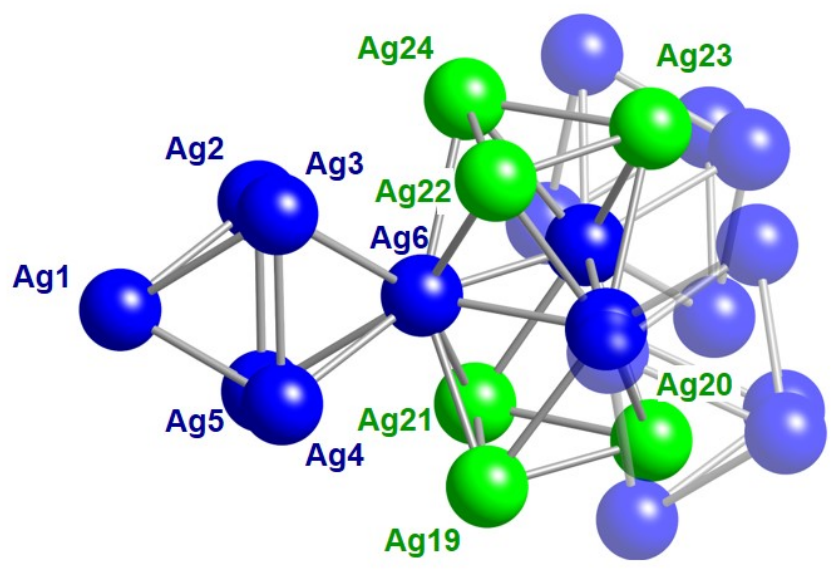

Figure S4. (a) Top view and (b) side view of the silver nanocluster $\left\{\mathrm{Ag}_{27}\right\}^{17+}$ of Ag27.
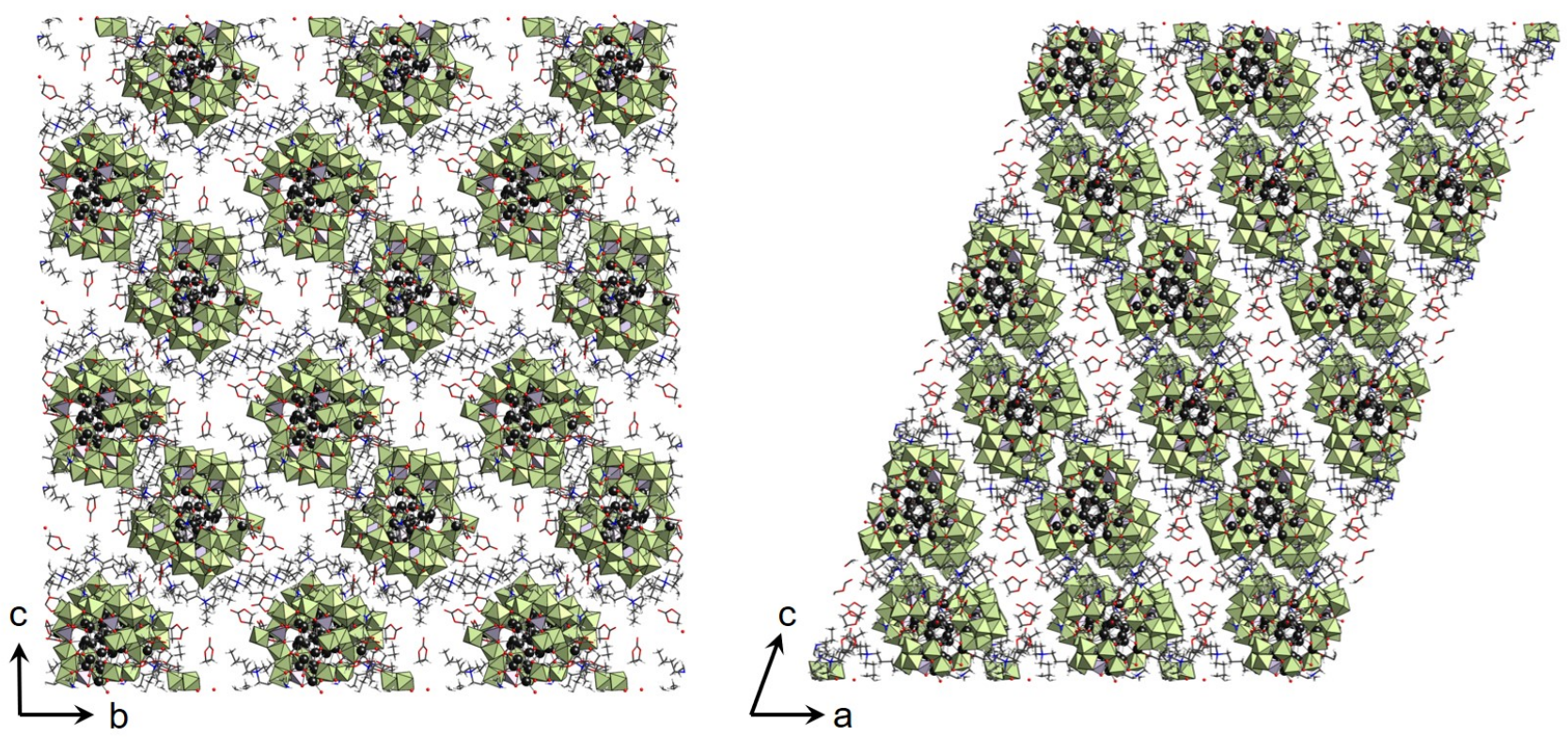

Figure S5. Crystal packings of Ag27. 


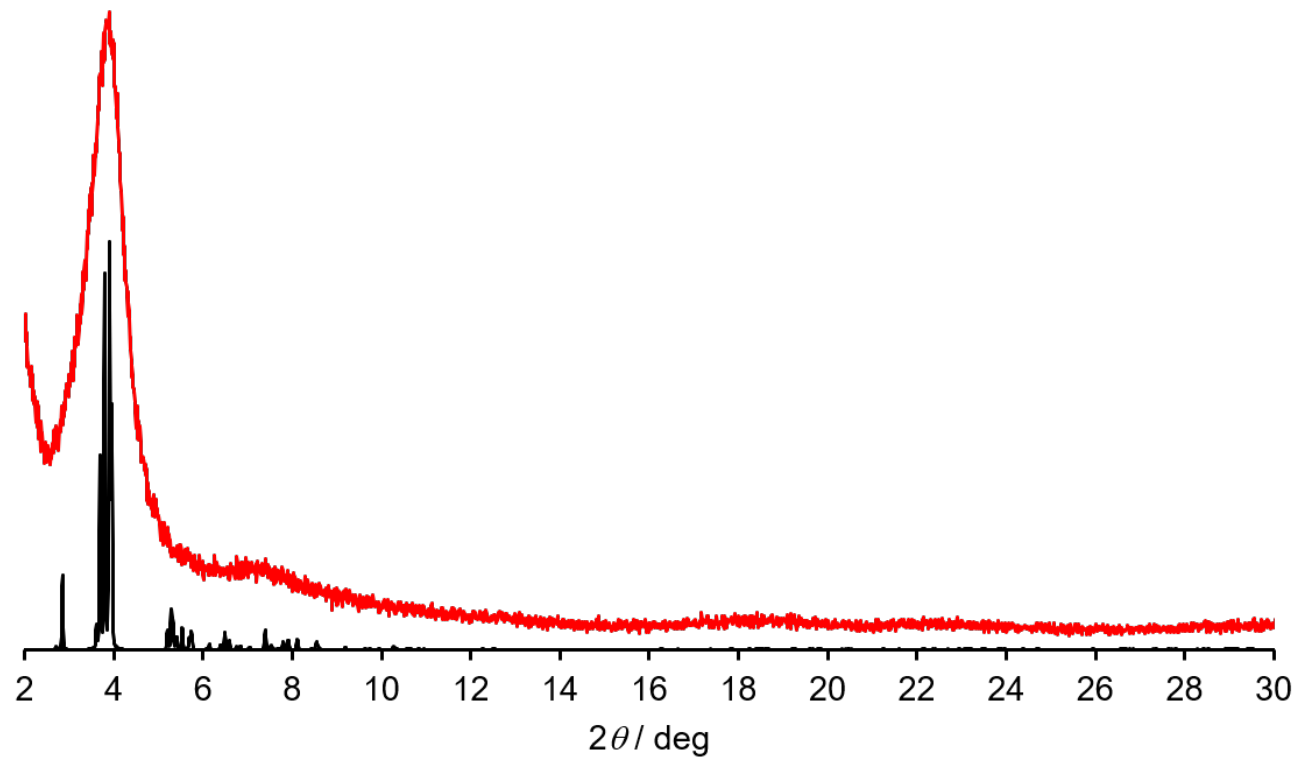

Figure S6. The observed (red line) and simulated (black line) powder XRD patterns of Ag27.

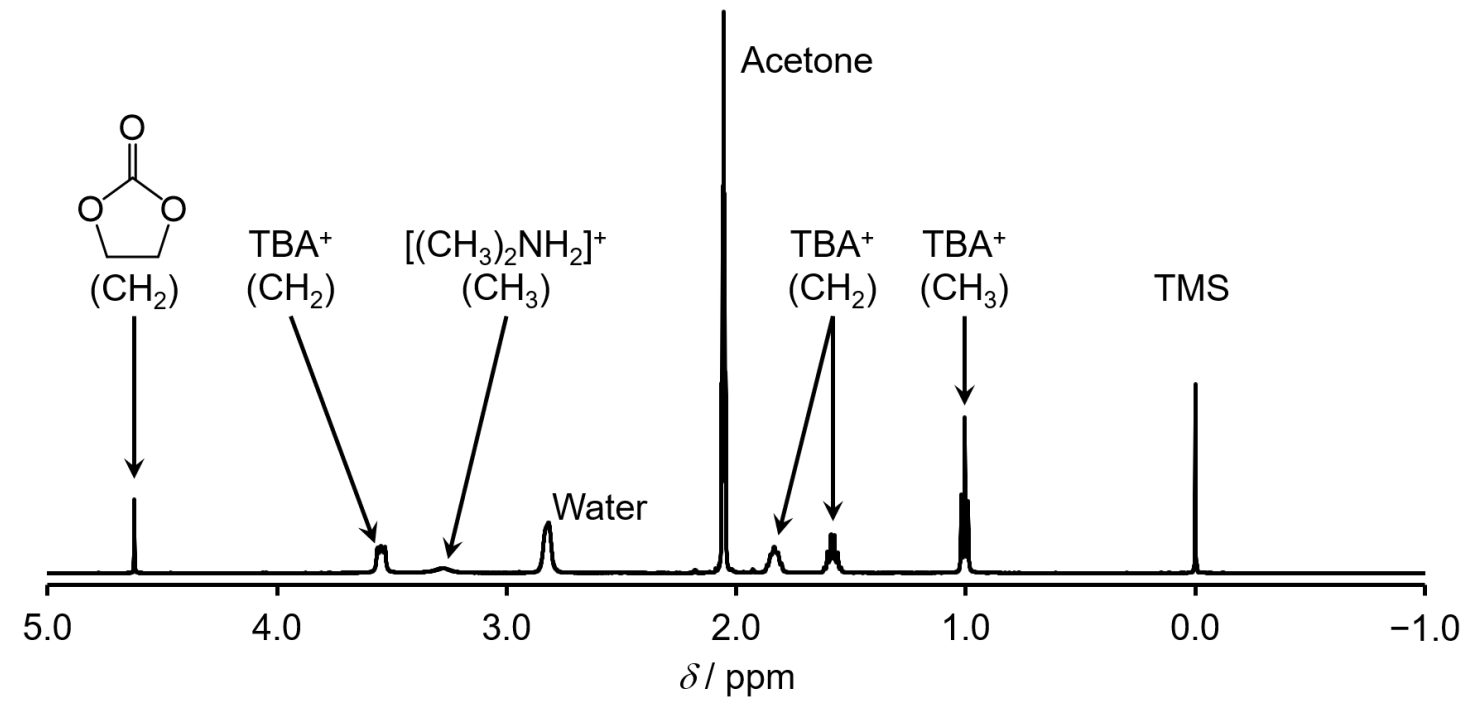

Figure S7. ${ }^{1} \mathrm{H}$ NMR spectrum of $\mathbf{A g 2 7}$ in acetone- $d_{6}$. 


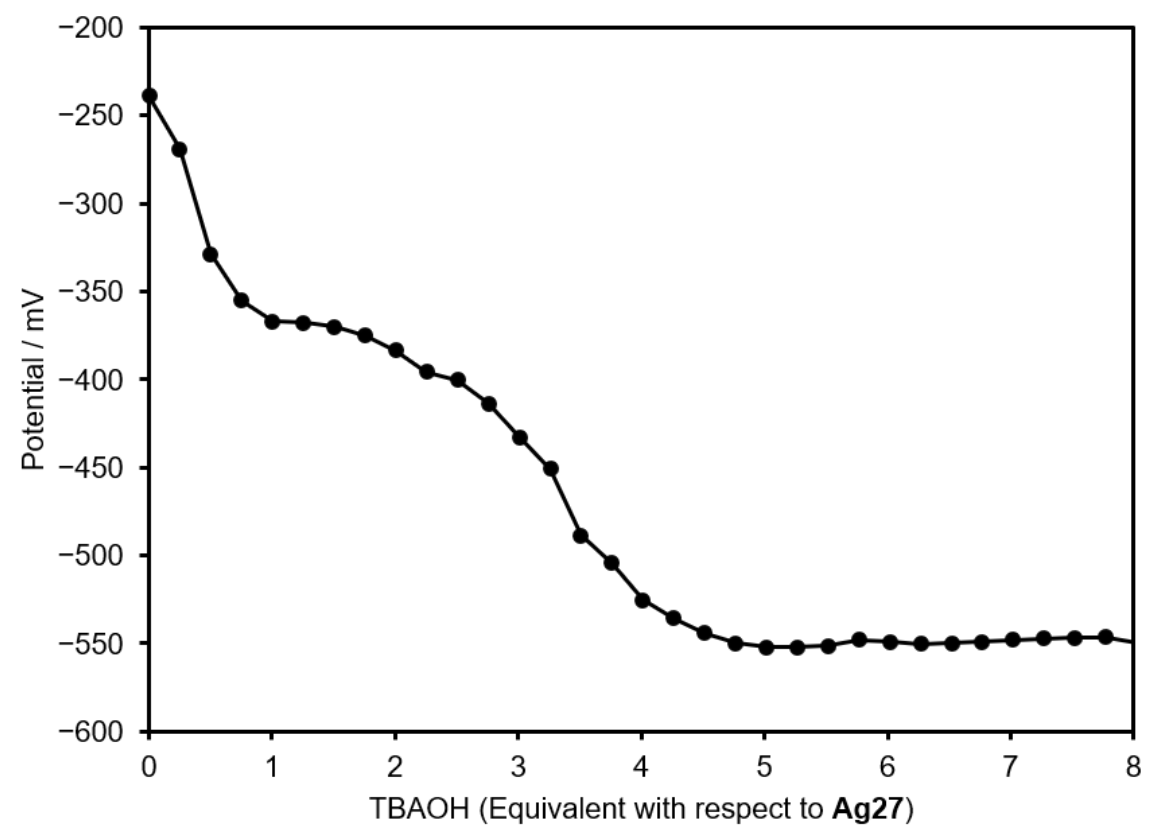

Figure S8. Profile for the potentiometric titration of Ag27 (18.9 $\mu \mathrm{mol})$ in acetonitrile with TBAOH (0.236 M, methanol solution) as titrant. The potentials are relative to a standard $\mathrm{Ag} / \mathrm{AgCl}$ electrode. 


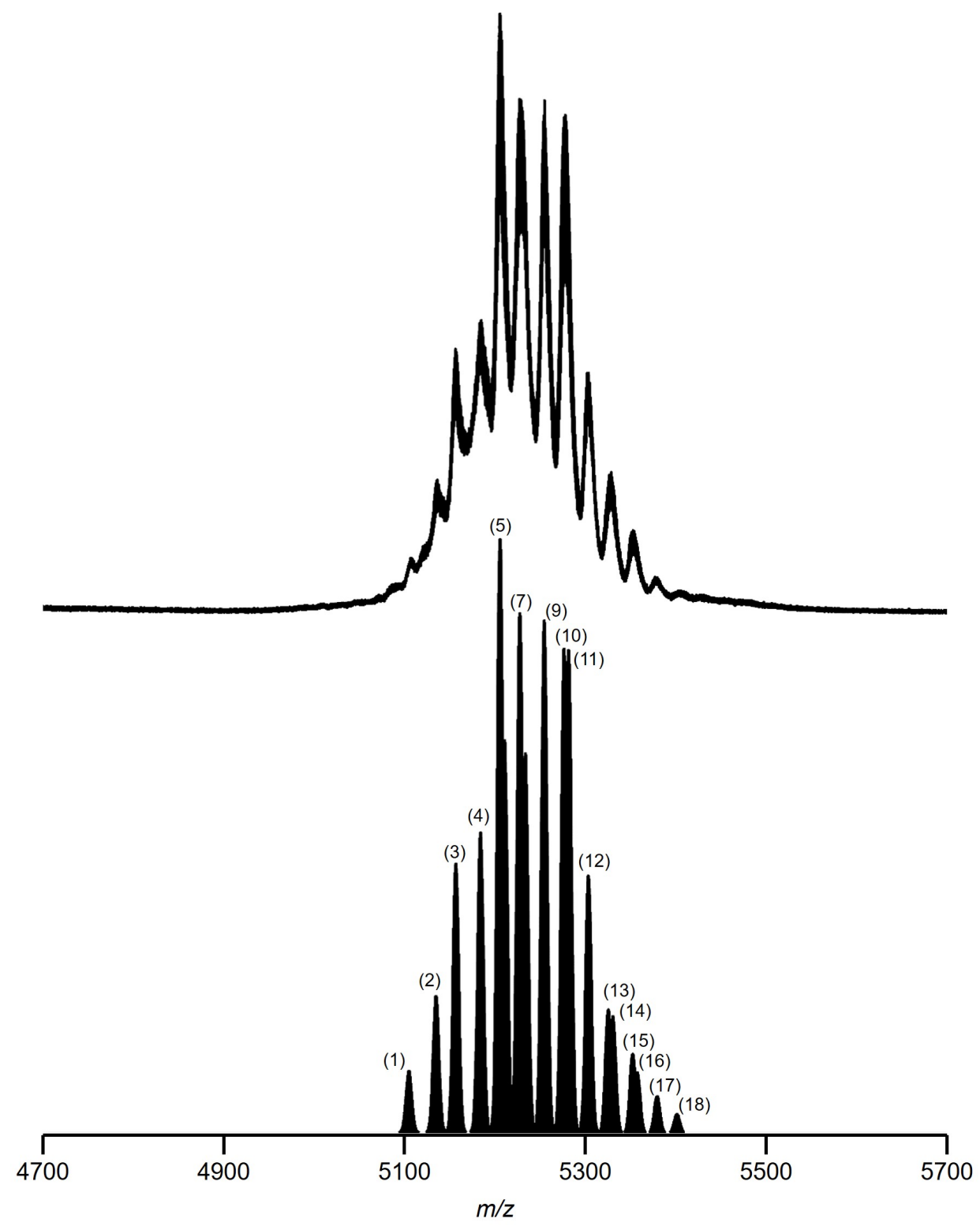

Figure S9. ESI-mass spectrum of Ag27 in acetone (top) and simulated patterns for $\left[\mathrm{TBA}_{m}\left(\mathrm{Me}_{2} \mathrm{NH}_{2}\right)_{22}\right.$ $\left.{ }_{m} \mathrm{H}_{11} \mathrm{Ag}_{29}\left(\mathrm{Si}_{2} \mathrm{~W}_{18} \mathrm{O}_{66}\right)_{3}\left(\mathrm{C}_{3} \mathrm{H}_{4} \mathrm{O}_{3}\right)_{n}\right]^{4+}(m=14-20, n=1-4$; bottom). Assignments of the simulated patterns $(m / z)$ are as follows:

(1) $\left[\mathrm{TBA}_{14}\left(\mathrm{Me}_{2} \mathrm{NH}_{2}\right)_{8} \mathrm{H}_{11} \mathrm{Ag}_{22}\left(\mathrm{Si}_{2} \mathrm{~W}_{18} \mathrm{O}_{66}\right)_{3}\left(\mathrm{C}_{3} \mathrm{H}_{4} \mathrm{O}_{3}\right)_{3}\right]^{4+}(5107.528) ;$; 2 [ $\left[\mathrm{TBA}_{15}\left(\mathrm{Me}_{2} \mathrm{NH}_{2}\right)_{7} \mathrm{H}_{11} \mathrm{Ag}_{22}\left(\mathrm{Si}_{2} \mathrm{~W}_{18} \mathrm{O}_{66}\right)_{3}\left(\mathrm{C}_{3} \mathrm{H}_{4} \mathrm{O}_{3}\right)_{2}\right]^{4+}(5134.579)$;

(3) $\left[\mathrm{TBA}_{15}\left(\mathrm{Me}_{2} \mathrm{NH}_{2}\right)_{7} \mathrm{H}_{11} \mathrm{Ag}_{22}\left(\mathrm{Si}_{2} \mathrm{~W}_{18} \mathrm{O}_{66}\right)_{3}\left(\mathrm{C}_{3} \mathrm{H}_{4} \mathrm{O}_{3}\right)_{3}\right]^{4+}(5156.583) ;$; (4) $\left[\mathrm{TBA}_{16}\left(\mathrm{Me}_{2} \mathrm{NH}_{2}\right)_{6} \mathrm{H}_{11} \mathrm{Ag}_{22}\left(\mathrm{Si}_{2} \mathrm{~W}_{18} \mathrm{O}_{66}\right)_{3}\left(\mathrm{C}_{3} \mathrm{H}_{4} \mathrm{O}_{3}\right)_{2}\right]^{4+}(5183.884)$;

(5) $\left[\mathrm{TBA}_{16}\left(\mathrm{Me}_{2} \mathrm{NH}_{2}\right)_{6} \mathrm{H}_{11} \mathrm{Ag}_{22}\left(\mathrm{Si}_{2} \mathrm{~W}_{18} \mathrm{O}_{66}\right)_{3}\left(\mathrm{C}_{3} \mathrm{H}_{4} \mathrm{O}_{3}\right)_{3}\right]^{4+}$ (5205.888); (6) [ $\left.\mathrm{TBA}_{17}\left(\mathrm{Me}_{2} \mathrm{NH}_{2}\right)_{5} \mathrm{H}_{11} \mathrm{Agg}_{22}\left(\mathrm{Si}_{2} \mathrm{~W}_{18} \mathrm{O}_{66}\right) 3\left(\mathrm{C}_{3} \mathrm{H}_{4} \mathrm{O}_{3}\right)\right]^{4+}$ (5210.935);

(7) $\left[\mathrm{TBA}_{16}\left(\mathrm{Me}_{2} \mathrm{NH}_{2}\right)_{6} \mathrm{H}_{11} \mathrm{Ag}_{22}\left(\mathrm{Si}_{2} \mathrm{~W}_{18} \mathrm{O}_{66}\right)_{3}\left(\mathrm{C}_{3} \mathrm{H}_{4} \mathrm{O}_{3}\right)_{4}\right]^{4+}(5227.892) ;$; 8 [ $\left.\mathrm{TBA}_{17}\left(\mathrm{Me}_{2} \mathrm{NH}_{2}\right)_{5} \mathrm{H}_{11} \mathrm{Ag}_{22}\left(\mathrm{Si}_{2} \mathrm{~W}_{18} \mathrm{O}_{66}\right)_{3}\left(\mathrm{C}_{3} \mathrm{H}_{4} \mathrm{O}_{3}\right)_{2}\right]^{4+}(5232.939)$;

(9) $\left[\mathrm{TBA}_{17}\left(\mathrm{Me}_{2} \mathrm{NH}_{2}\right)_{5} \mathrm{H}_{11} \mathrm{Ag}_{22}\left(\mathrm{Si}_{2} \mathrm{~W}_{18} \mathrm{O}_{66}\right)_{3}\left(\mathrm{C}_{3} \mathrm{H}_{4} \mathrm{O}_{3}\right)_{3}\right]^{4+}$ (5254.942); (10) [ $\left.\left.\mathrm{TBA}_{17}\left(\mathrm{Me}_{2} \mathrm{NH}_{2}\right)_{5} \mathrm{H}_{11} \mathrm{Ag}_{22}\left(\mathrm{Si}_{2} \mathrm{~W}_{18} \mathrm{O}_{66}\right)_{3}\left(\mathrm{C}_{3} \mathrm{H}_{4} \mathrm{O}_{3}\right)\right]_{4}\right]^{4+}(5276.947)$;

(11) $\left[\mathrm{TBA}_{18}\left(\mathrm{Me}_{2} \mathrm{NH}_{2}\right)_{4} \mathrm{H}_{11} \mathrm{Ag}_{22}\left(\mathrm{Si}_{2} \mathrm{~W}_{18} \mathrm{O}_{66}\right)_{3}\left(\mathrm{C}_{3} \mathrm{H}_{4} \mathrm{O}_{3}\right)_{2}\right]^{4+}$ (5281.994); (12) $\left[\mathrm{TBA}_{18}\left(\mathrm{Me}_{2} \mathrm{NH}_{2}\right)_{4} \mathrm{H}_{11} \mathrm{Ag}_{29}\left(\mathrm{Si}_{2} \mathrm{~W}_{18} \mathrm{O}_{66}\right)_{3}\left(\mathrm{C}_{3} \mathrm{H}_{4} \mathrm{O}_{3}\right)_{3}\right]^{4+}(5303.998)$;

(13) $\left[\mathrm{TBA}_{18}\left(\mathrm{Me}_{2} \mathrm{NH}_{2}\right)_{4} \mathrm{H}_{11} \mathrm{Ag}_{22}\left(\mathrm{Si}_{2} \mathrm{~W}_{18} \mathrm{O}_{66}\right)_{3}\left(\mathrm{C}_{3} \mathrm{H}_{4} \mathrm{O}_{3}\right)_{4}\right]^{4+}$ (5326.002); (14) $\left[\mathrm{TBA}_{19}\left(\mathrm{Me}_{2} \mathrm{NH}_{2}\right)_{3} \mathrm{H}_{11} \mathrm{Agg}_{22}\left(\mathrm{Si}_{2} \mathrm{~W}_{18} \mathrm{O}_{66}\right)_{3}\left(\mathrm{C}_{3} \mathrm{H}_{4} \mathrm{O}_{3}\right)_{2}\right]^{4+}(331.049)$;

(15) $\left[\mathrm{TBA}_{19}\left(\mathrm{Me}_{2} \mathrm{NH}_{2}\right)_{3} \mathrm{H}_{11} \mathrm{Ag}_{22}\left(\mathrm{Si}_{2} \mathrm{~W}_{18} \mathrm{O}_{66}\right)_{3}\left(\mathrm{C}_{3} \mathrm{H}_{4} \mathrm{O}_{3}\right)_{3}\right]^{4+}$ (5353.053); (16) $\left[\mathrm{TBA}_{20}\left(\mathrm{Me}_{2} \mathrm{NH}_{2}\right)_{2} \mathrm{H}_{11} \mathrm{Ag}_{22}\left(\mathrm{Si}_{2} \mathrm{~W}_{18} \mathrm{O}_{66}\right)_{3}\left(\mathrm{C}_{3} \mathrm{H}_{4} \mathrm{O}_{3}\right)\right]^{4+}(5358.100)$;

(17) $\left[\mathrm{TBA}_{20}\left(\mathrm{Me}_{2} \mathrm{NH}_{2}\right)_{2} \mathrm{H}_{11} \mathrm{Ag}_{22}\left(\mathrm{Si}_{2} \mathrm{~W}_{18} \mathrm{O}_{66}\right)_{3}\left(\mathrm{C}_{3} \mathrm{H}_{4} \mathrm{O}_{3}\right)_{2}\right]^{4+}$ (5380.104); (18) [ $\left.\mathrm{TBA}_{20}\left(\mathrm{Me}_{2} \mathrm{NH}_{2}\right)_{2} \mathrm{H}_{11} \mathrm{Agg}_{20}\left(\mathrm{Si}_{2} \mathrm{~W}_{18} \mathrm{O}_{66}\right)_{3}\left(\mathrm{C}_{3} \mathrm{H}_{4} \mathrm{O}_{3}\right)_{3}\right]^{4+}(5402.107)$; 
(a)

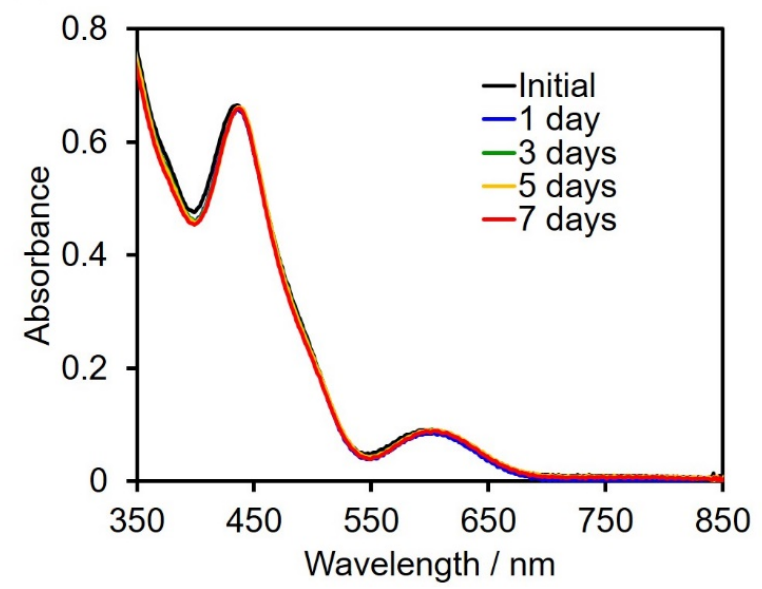

(c)

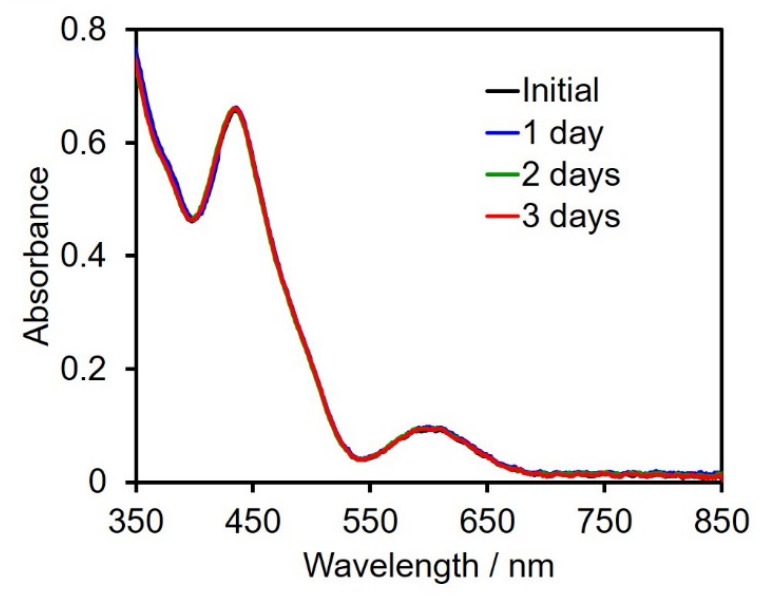

(b)

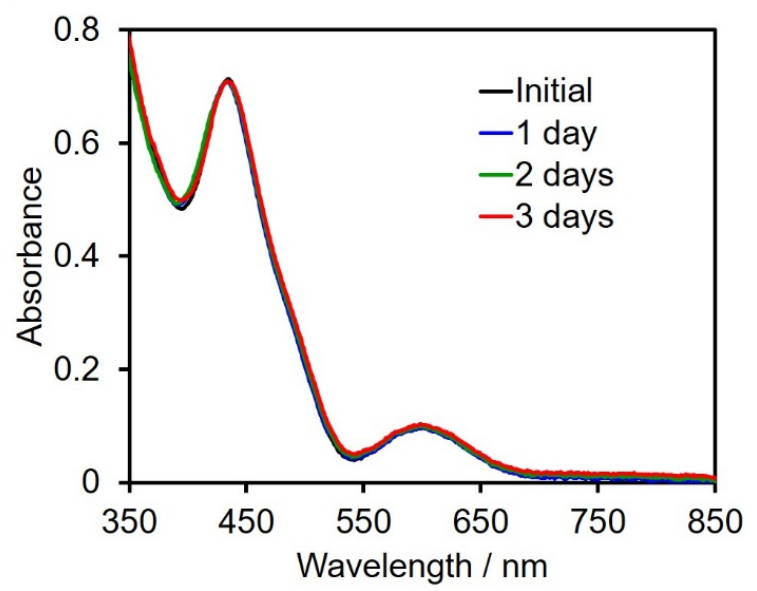

Figure S10. UV-Vis spectra of Ag27 (10 $\mu \mathrm{M})$ in (a) acetone containing ethylene carbonate (1 M), (b) acetone, and (c) acetonitrile. 
(a)

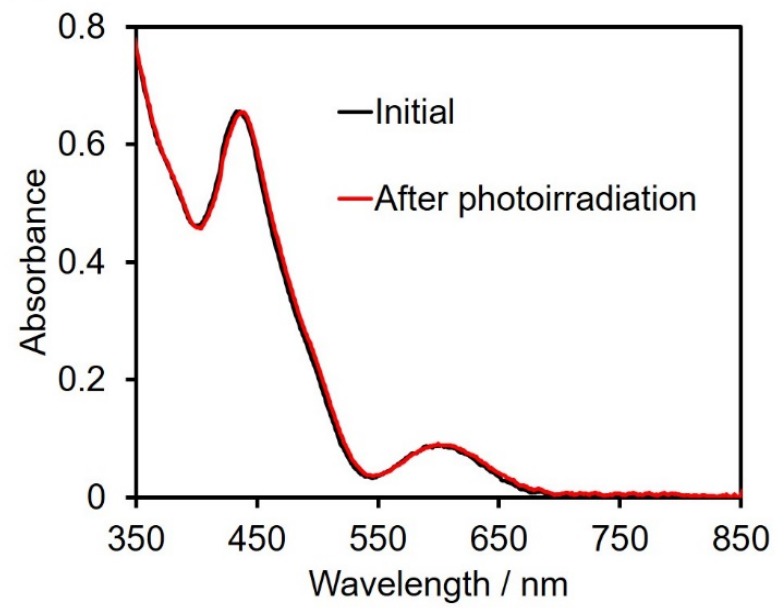

(b)

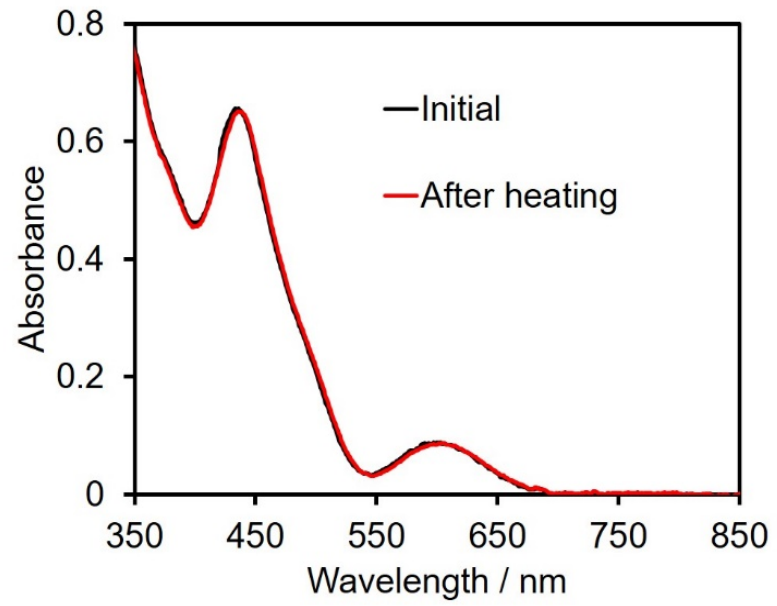

Figure S11. UV-Vis spectra of Ag27 $(10 \mu \mathrm{M})$ in acetonitrile before and after (a) photoirradiation $(\lambda>400 \mathrm{~nm}$, $300 \mathrm{~W}$ Xe lamp) for $6 \mathrm{~h}$ and (b) heating at $60^{\circ} \mathrm{C}$ for $6 \mathrm{~h}$. 


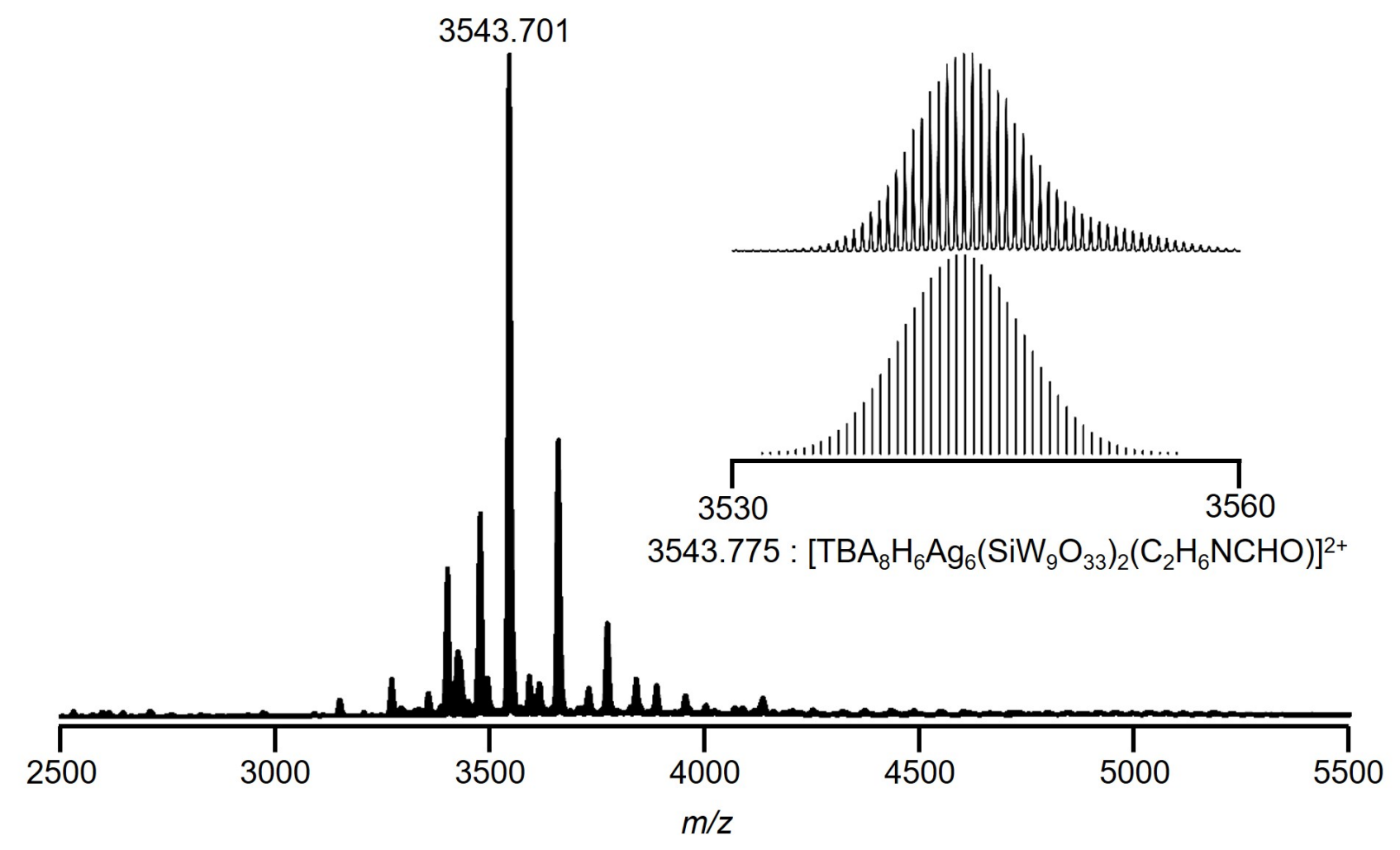

Figure S12. ESI-mass spectrum of the synthetic solution of Ag27. Insets: spectrum in the range of $\mathrm{m} / \mathrm{z} 3530$ 3560 , and simulated pattern for $\left[\mathrm{TBA}_{8} \mathrm{H}_{6} \mathrm{Ag}_{6}\left(\mathrm{SiW}_{9} \mathrm{O}_{33}\right)_{2}(\mathrm{DMF})\right]^{2+}(\mathrm{m} / z$ 3543.775). 
(a)

W based orbitals (LUMO - LUMO+1)
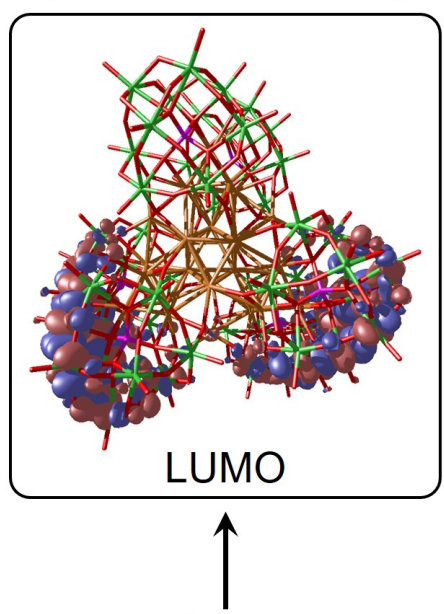

$\left\{\mathrm{Ag}_{27}\right\}^{17+}$-to-POM

Charge Transfer

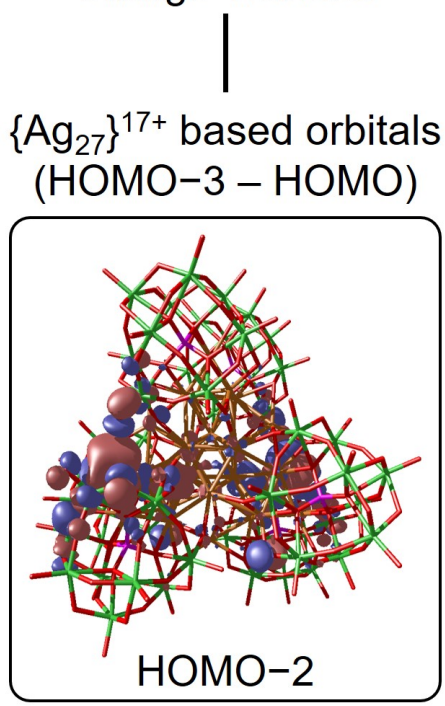

(b)

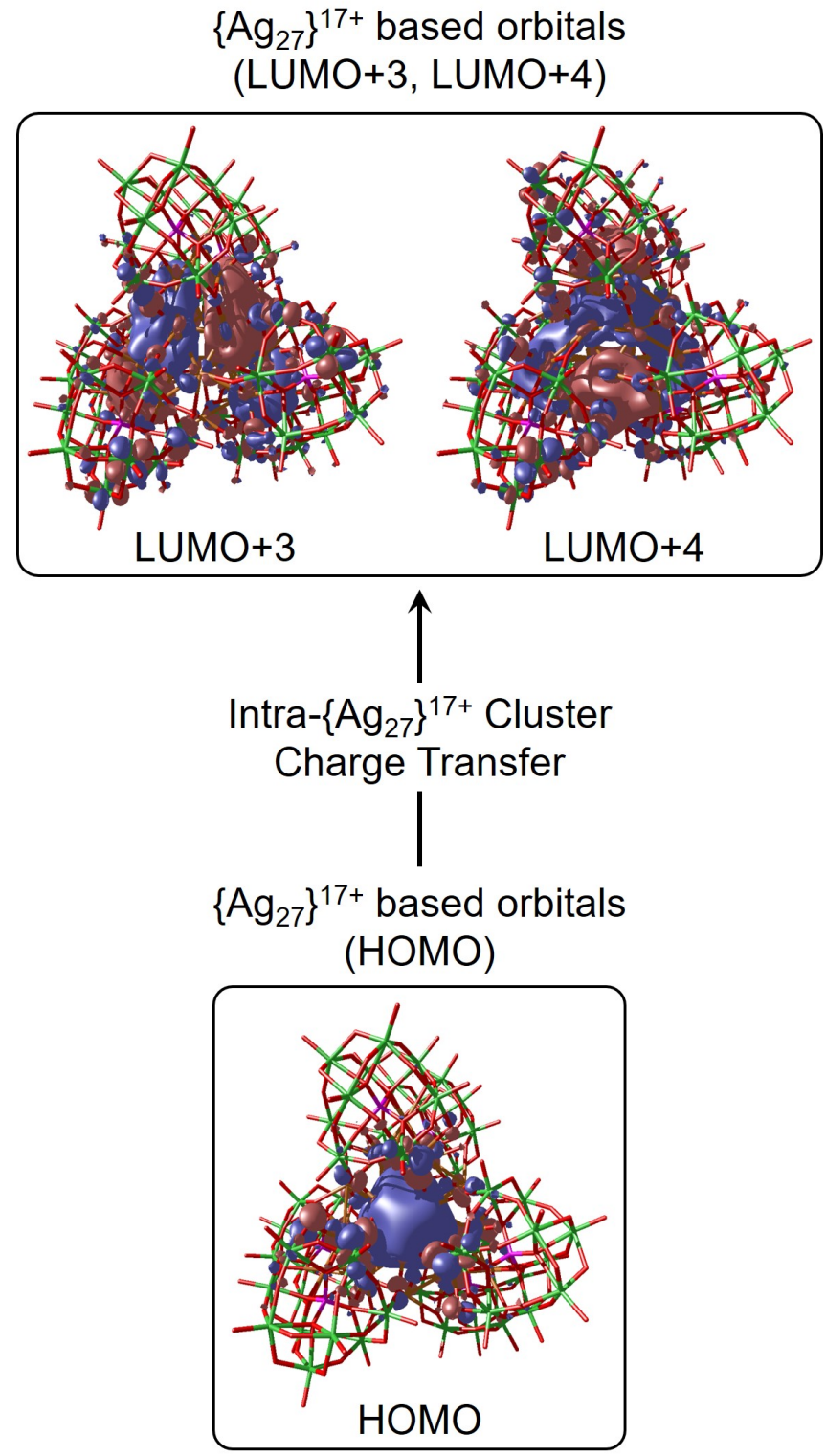

Figure S13. Two types of possible visible-light-induced charge transfer on Ag27 based on the TD-DFT calculation. (a) $\left\{\mathrm{Ag}_{27}\right\}^{17+}$ nanocluster core to POM ligands charge transfer and (b) Inter- $\left\{\mathrm{Ag}_{27}\right\}^{17+}$ core charge transfer. 


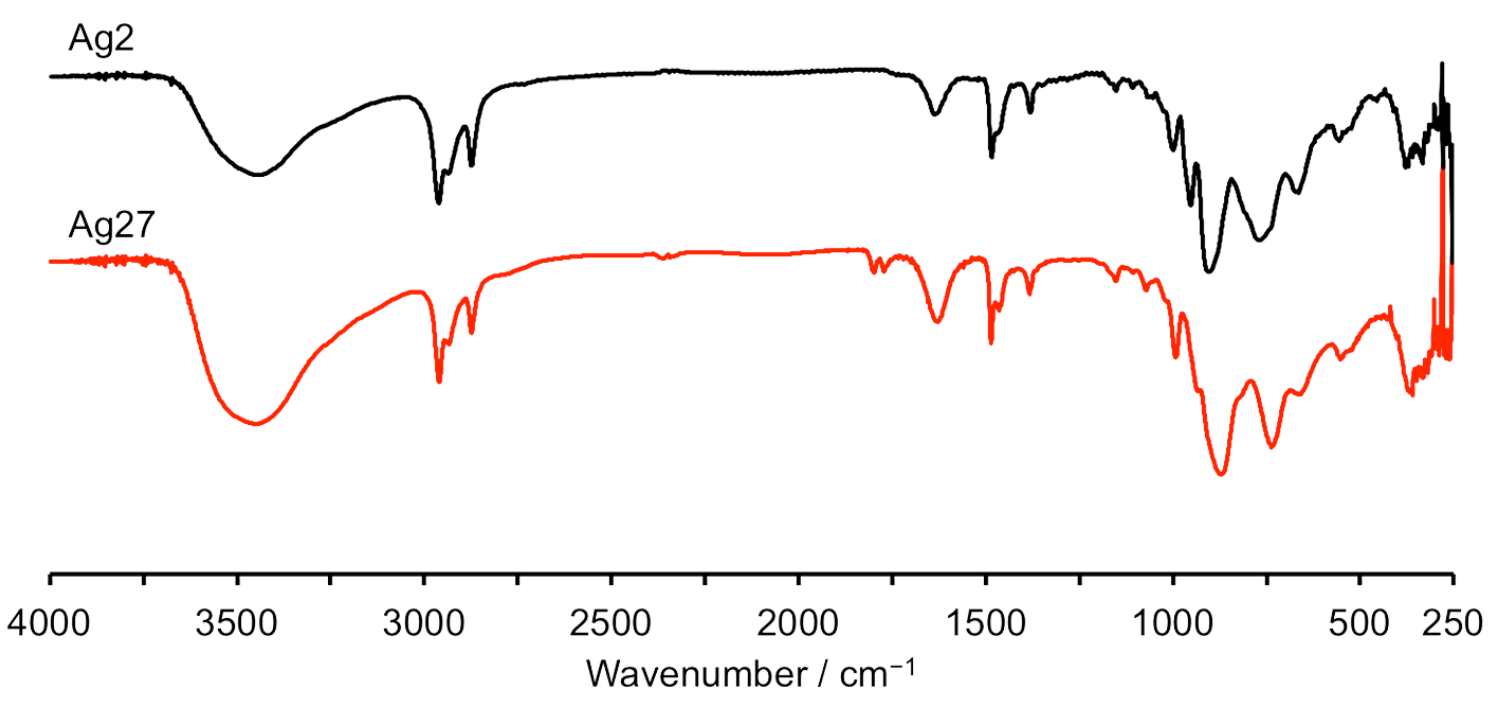

Figure S14. IR spectra of Ag2 and Ag27.

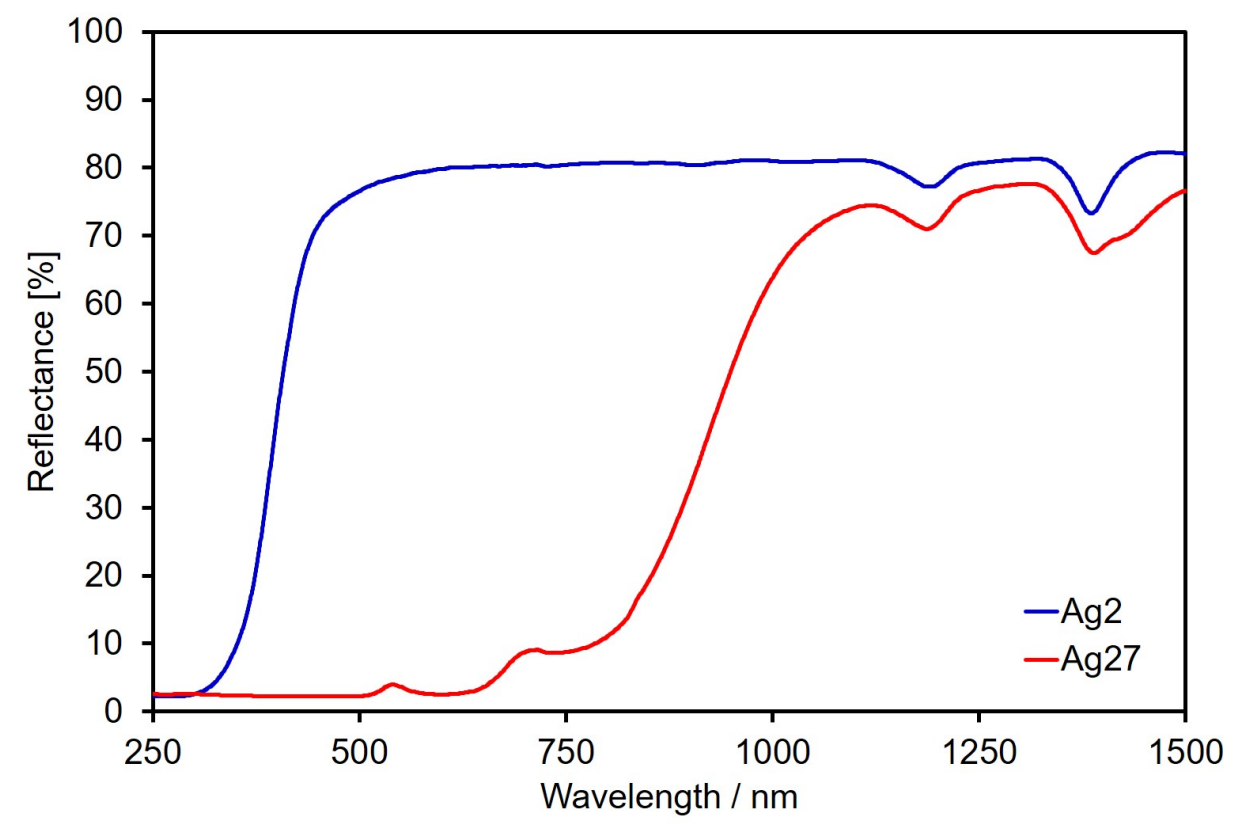

Figure S15. Diffuse reflectance UV-Vis spectra of Ag2 and Ag27. 


\section{References}

(S1) Minato, T.; Suzuki, K.; Kamata, K.; Mizuno, N. Synthesis of $\alpha$-Dawson-type silicotungstate $\left[\alpha-\mathrm{Si}_{2} \mathrm{~W}_{18} \mathrm{O}_{62}\right]^{8-}$ and protonation and deprotonation inside the aperture through intramolecular hydrogen bonds. Chem. Eur. J. 2014, 20, 5946-5952.

(S2) Minato, T.; Suzuki, K.; Yamaguchi, K.; Mizuno, N. Alkoxides of trivacant lacunary polyoxometalates. Chem. Eur. J. 2017, 23, 14213-14220.

(S3) Rigaku OD. CrysAlis PRO. Rigaku Oxford Diffraction Ltd, Yarnton, England (2018).

(S4) Farrugia, L. J. WinGX suite for small-molecule single-crystal crystallography. J. Appl. Crystallogr. 1999, 32, 837-838.

(S5) (a) Sheldrick, G. M. A short history of SHELX. Acta Cryst. 2008, A64, 112-122. (b) Sheldrick, G. M. Crystal structure refinement with SHELXL. Acta Cryst. 2015, C71, 3-8.

(S6) van der Sluis, P.; Spek, A. L. BYPASS: An effective method for the refinement of crystal structures containing disordered solvent regions. Acta Crystallogr. 1990, A46, 194.

Brese, N. E.; O’Keeffe, M. Bond-valence parameters for solids. Acta Crystallogr. Sect. 1991, B47, $192-197$.

(S8) Yang, H.; Lei, J.; Wu, B.; Wang, Y.; Zhou, M.; Xia, A.; Zheng, L.; Zheng, N. Crystal structure of a luminescent thiolated $\mathrm{Ag}$ nanocluster with an octahedral $\mathrm{Ag}_{6}{ }^{4+}$ core. Chem. Commun. 2013, 49, 300302.

(S9) Shen, X.-T.; Ma, X.-L.; Ni, Q.-L.; Ma, M.-X.,; Gui, L.-C.; Hou, C.; Hou, R.-B.; Wang, X.-J. $\left[\mathrm{Ag}_{15}(\mathrm{~N} \text {-triphos })_{4}\left(\mathrm{Cl}_{4}\right)\right]\left(\mathrm{NO}_{3}\right)_{3}$ : a stable $\mathrm{Ag}-\mathrm{P}$ superatom with eight electrons $(\mathrm{N}$-triphos $=$ tris((diphenylphosphino) methyl)amine). Nanoscale 2018, 10, 515-519.

(S10) Yang, H.; Wang, Y.; Zheng, N. Stabilizing subnanometer $\operatorname{Ag}(0)$ nanoclusters by thiolate and diphosphine ligands and their crystal structures. Nanoscale 2013, 5, 2674-2677.

(S11) Yuan, S.-F.; Li, P.; Tang, Q.; Wan, X.-K.; Jiang, D.; Wang, Q.-M. Alkynyl-protected silver nanoclusters featuring an anticuboctahedral kernel. Nanoscale 2017, 9, 11405-11409.

(S12) Dhayal, R. S.; Lin, Y.-R.; Liao, J.-H.; Chen, Y.-J.; Liu, Y.-C.; Chiang, M.-H.; Kahlal, S.; Saillard, 
J.-Y.; Liu, C. W. $\left[\operatorname{Ag}_{20}\left\{\mathrm{~S}_{2} \mathrm{P}(\mathrm{OR})_{2}\right\}_{12}\right]$ : A superatom complex with a chiral metallic core and high potential for isomerism. Chem. Eur. J. 2016, 22, 9943-9947.

(S13) Chang, W.-T.; Lee, P.-Y.; Liao, J.-H.; Chakahari, K. K.; Kahlal, S.; Liu, Y.-C.; Chiang, M.-H.; Saillard, J.-Y.; Liu, C. W. Eight-electron silver and mixed gold/silver nanoclusters stabilized by selenium donor ligands. Angew. Chem., Int. Ed. 2017, 56, 10178-10182.

(S14) Dhayal, R. S.; Liao, J.-H.; Liu, Y.-C.; Chiang, M.-H.; Kahlal, S.; Saillard, J.-Y.; Liu, C. W. $\left[\mathrm{Ag}_{21}\left\{\mathrm{~S}_{2} \mathrm{P}\left(\mathrm{O}^{i} \mathrm{Pr}\right)_{2}\right\}_{12}\right]^{+}:$An eight electron superatom. Angew. Chem., Int. Ed. 2015, 54, 3702-3706.

(S15) Liu, C.; Li, T.; Abroshan, H.; Li, Z.; Zhang, C.; Kim, H. J.; Li, G.; Jin, R. Chiral Ag 23 nanocluster with open shell electronic structure and helical face-centered cubic framework. Nat. Commun. 2018, 9, 744.

(S16) Joshi, C. P.; Bootharaju, M. S.; Alhilaly, M. J.; Bakr, O. M. $\left[\operatorname{Ag}_{25}(\mathrm{SR})_{18}\right]^{-}$: The "golden" silver nanoparticle. J. Am. Chem. Soc. 2015, 137, 11578-11581.

(S17) Xi, X.-J.; Yang. J.-S.; Wang, J.-Y.; Dong, X.-Y.; Zang, S.-Q. New stable isomorphous Ag $_{34}$ and $\mathrm{Ag}_{33} \mathrm{Au}$ nanoclusters with an open shell electronic structure. Nanoscale 2015, 10, 21013-21018.

(S18) Sun, D.; Luo, G.-G.; Zhang, N.; Huang, R.-B.; Zheng, L.-S. Simultaneous self-assembly of a cage-like silver(I) complex encapsulating an $\mathrm{Ag}_{6}$ neutral cluster core and carbon dioxide fixation. Chem. Commun. 2011, 47, 1461-1463.

(S19) Guan, Z.-J.; Zeng, J.-L.; Nan, Z.-A.; Wan, X.-K.; Lin, Y.-M.; Wang, Q.-M. Thiacalix[4]arene: new protection for metal nanoclusters. Sci. Adv. 2016, 2, e1600323.

(S20) Chai, J.; Yang, S.; Ying, L.; Chen, T.; Wang, S.; Yu, H.; Zhu, M. A Unique Pair: $\operatorname{Ag}_{40}$ and $\operatorname{Ag}_{46}$ nanoclusters with the same surface but different cores for structure-property correlation. J. Am. Chem. Soc. 2018, 140, 15582-15585.

(S21) Yuan, X.; Sun, C.; Li, X.; Malola, S.; Teo, B. K.; Häkkinen, H.; Zheng, L.-S.; Zheng, N. Combinatorial identification of hydrides in a ligated $\mathrm{Ag}_{40}$ nanocluster with noncompact metal core. $J$. Am. Chem. Soc. 2019, 141, 11905-11911.

(S22) Yang, H.; Wang, Y.; Huang, H.; Gell, L.; Lehtovaara, L.; Malona, S.; Häkkeinen, H.; Zheng, N. 
All-thiol-stabilized $\mathrm{Ag}_{44}$ and $\mathrm{Au}_{12} \mathrm{Ag}_{32}$ nanoparticles with single-crystal structures. Nat. Commun. 2013, 4, 2422.

Desireddy, A.; Conn, B. E.; Guo, J.; Yoon, J.; Barnett, R. N.; Monahan, B. M.; Kirschbaum, K.; Griffith, W. P.; Whetten, R. L.; Landman, U.; Bigioni, T. P. Ultrastable silver nanoparticles. Nature 2013, 501, 399-402.

(S24) Zhang, S.-S.; Alkan, F.; Su, H.-F.; Aikens, C. M.; Tung, C.-H.; Sun, D. [Ag $\left.{ }_{48}\left(\mathrm{C} \equiv \mathrm{C}^{t} \mathrm{Bu}\right)_{20}\left(\mathrm{CrO}_{4}\right)_{7}\right]$ : an atomically precise silver nanocluster co-protected by inorganic and organic ligands. J. Am. Chem. Soc. 2019, $141,4460-4467$.

(S25) Du, W.; Jin, S.; Xiong, L.; Chen, M.; Zhang, J.; Zou, X.; Pei, Y.; Wang, S.; Zhu, M. $\mathrm{Ag}_{50}(\mathrm{Dppm})_{6}(\mathrm{SR})_{30}$ and its homologue $\operatorname{Au}_{x} \mathrm{Ag}_{50-x}(\mathrm{Dppm})_{6}(\mathrm{SR})_{30}$ alloy nanocluster: seeded growth, structure determination, and differences in properties. J. Am. Chem. Soc. 2017, 139, 1618-1624. Duan, G.-X.; Tian, L.; Wen, J.-B.; Li, L.-Y.; Xie, Y.-P.; Lu, X. An atomically precise all-tert-butylethynideprotected $\mathrm{Ag}_{51}$ superatom nanocluster with color tunability. Nanoscale 2018, 10, 18915-18919.

(S27) Jin, S.; Wang, S.; Song, Y.; Zhou, M.; Zhong, J.; Zhang, J.; Xia, A.; Pei, Y.; Chen, M.; Li, P.; Zhu, M. Crystal structure and optical properties of the $\left[\mathrm{Ag}_{62} \mathrm{~S}_{12}\left(\mathrm{SBu}^{t}\right)_{32}\right]^{2+}$ nanocluster with a complete face-centered cubic kernel. J. Am. Chem. Soc. 2017, 139, 1618-1624.

(S28) Alhilaly, M. J.; Bootharaju, M. S.; Joshi, C. P.; Besong, T. M.; Emwas, A.-H.; Juarez-Mosqueda, R.; Kaappa, S.; Malola, S.; Adil, K.; Shkurenko, A.; Häkkinen, H.; Eddaoudi, M.; Bakr, O. M. $\left[\mathrm{Ag}_{67}\left(\mathrm{SPhMe}_{2}\right)_{32}\left(\mathrm{PPh}_{3}\right)_{8}\right]^{3+}:$ Synthesis, total structure, and optical properties of a large box-shaped silver nanocluster. J. Am. Chem. Soc. 2016, 138, 14727-14732.

Qu, M.; Li, H.; Xie, L.-H.; Yan, S.-T.; Li, J.-R.; Wang, J.-H.; Wei, C.-Y.; Wu, Y.-W.; Zhang, X.-M. Bidentate phosphine-assisted synthesis of an all-alkynyl-protected $\mathrm{Ag}_{74}$ nanocluster. J. Am. Chem. Soc. 2017, 139, 12346-12349.

(S30) Yang. H.; Yan, J.; Wang, Y.; Deng, G.; Su, H.; Zhao, X.; Xu, C.; Teo, B. K.; Zheng, N. From racemic metal nanoparticles to optically pure enantiomers in one pot. J. Am. Chem. Soc. 2017, 139, 16113- 
16116.

(S31) Ren, L.; Yuan, P.; Su, H.; Malola, S.; Lin, S.; Tang, Z.; Teo, B. K.; Häkkinen, H.; Zheng, L.; Zheng, N. Bulky surface ligands promote surface reactivities of $\left[\mathrm{Ag}_{141} \mathrm{X}_{12}(\mathrm{SAdm})_{40}\right]^{3+}(\mathrm{X}=\mathrm{Cl}, \mathrm{Br}, \mathrm{I})$ nanoclusters: models for multiple-twinned nanoparticles. J. Am. Chem. Soc. 2017, 139, 12288-13291.

(S32) Song, Y.; Lambright, K.; Zhou, M.; Kirschbaum, K.; Xiang, J.; Xia, A.; Zhu, M.; Jin, R. Large-scale synthesis, crystal structure, and optical properties of the $\mathrm{Ag}_{146} \mathrm{Br}_{2}(\mathrm{SR})_{80}$ nanocluster. ACS Nano 2018 , $12,9318-9325$.

(S33) Yan, J.; Zhang, J.; Chen, X.; Malola, S.; Zhou, B.; Selenius, E.; Zhang, X.; Yuan, P.; Deng, G.; Liu, K.; Su, H.; Teo, B. K.; Häkkinen, H.; Zheng, L.; Zheng, N. Thiol-stabilized atomically precise, superatomic silver nanoparticles for catalysing cycloisomerization of alkynyl amines. National Science Review 2018, 5, 694-702.

(S34) Liu, J.-Y.; Alkan, F.; Wang, Z.; Zhang, Z.-Y.; Kurmoo, M.; Yan, Z.; Zhao, Q.-Q.; Aikens, C. M.; Tung, C.-H.; Sun, D. Different silver nanoparticles in one crystal: $\operatorname{Ag}_{210}\left({ }^{i} \mathrm{PrPhS}\right)_{71}\left(\mathrm{Ph}_{3} \mathrm{P}\right)_{5} \mathrm{Cl}$ and $\mathrm{Ag}_{211}\left({ }^{i} \mathrm{PrPhS}\right)_{71}\left(\mathrm{Ph}_{3} \mathrm{P}\right)_{6} \mathrm{Cl}$. Angew. Chem., Int. Ed. 2019, 58, 195-199.

(S35) Yang, H.; Wang, Y.; Chen, X.; Zhao, X.; Gu, L.; Huang, H.; Yan, J.; Xu, C.; Li, G.; Wu, J.; Edwards, A. J.; Dittrich, B.; Tang, Z.; Wang, D.; Lehtovaara, L.; Häkkinen, H.; Zheng, N. Plasmonic twinned silver nanoparticles with molecular precision. Nat. Commun. 2016, 7, 12809.

(S36) Kikukawa, Y.; Kuroda, Y.; Suzuki, K.; Hibino, M.; Yamaguchi, K. Mizuno, N. A discrete octahedrally shaped $\left[\mathrm{Ag}_{6}\right]^{4+}$ cluster encapsulated within silicotungstate ligands. Chem. Commun. 2013, 49, 376$378(2013)$

(S37) Wang, Z.; Su, H.-F.; Kurmoo, M.; Tung, C.-H.; Sun. D.; Zheng, L.-S. Trapping an octahedral Ag $\operatorname{Ag}_{6}$ kernel in a seven-fold symmetric $\mathrm{Ag}_{56}$ nanowheel. Nat. Commun. 2018, 9, 2094.

(S38) Chen, S.; Fang, W.-H.; Zhang, L.; Zhang, J. Atomically precise multimetallic semiconductive nanoclusters with optical limiting effects. Angew. Chem., Int. Ed. 2018, 57, 11252-11256.

(S39) Su, Y.-M.; Wang, Z.; Zhuang, G.-L.; Zhao, Q.-Q.; Wang, X.-P.; Tung, C.-H.; Sun, D., Unusual fcc-structured $\mathrm{Ag}_{10}$ kernels trapped in $\mathrm{Ag}_{70}$ nanoclusters. Chem. Sci. 2019, 10, 564-568. 
(S40) Liu, J.-W.; Wang, Z.; Chai, Y.-M.; Kurmoo, M.; Zhao, Q.-Q.; Wang, X.-P.; Tung, C.-H.; Sun, D. Core modulation of 70-nuclei core-shell silver nanoclusters. Angew. Chem., Int. Ed. 2019, 58, 6276-6279. 